\title{
A Distributed Model Predictive Control for Multiple Mobile Robots with the Model Uncertainty
}

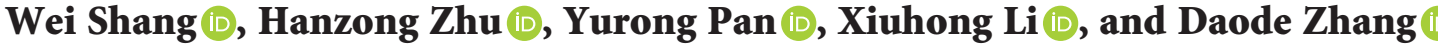 \\ College of Engineering Machinery, Hubei University of Technology, Wuhan 430068, China \\ Correspondence should be addressed to Yurong Pan; 375499678@qq.com
}

Received 1 April 2021; Revised 5 June 2021; Accepted 8 June 2021; Published 21 June 2021

Academic Editor: Ya Jia

Copyright (c) 2021 Wei Shang et al. This is an open access article distributed under the Creative Commons Attribution License, which permits unrestricted use, distribution, and reproduction in any medium, provided the original work is properly cited.

In this paper, a distributed predictive control with the model uncertainty which uses the data-driven strategy and robust theory (data-driven RDMPC) is proposed for the formation control of multiple mobile robots. The robust performance objective minimization is applied to replace the quadratic performance objective minimization to establish the optimization problem, where the model uncertainty is considered in the distributed system. The control policy is derived by applying the data-driven strategy, and the future predictive value is obtained by employing the linear law in the historical data. Lyapunov theory is referred to analyze the stability of the mobile robot formation system. The effectiveness of the proposed method is proved by a set of simulation experiments.

\section{Introduction}

In recent years, the robot formation control has become a key technology of multiple mobile robots' research studies which can be used in the military field, production field, and service field [1-4]. In contrast with a single mobile robot, multiple mobile robots have the advantages of coordination and cooperation, which could improve the efficiency of completing tasks. The multiple mobile robots also enhance the fault tolerance and robustness of the system and strengthen the ability of robots' environment recognition. Comparing with the leader-follower method [5-8], behavior-based method [9-11], and virtual structure method $[12,13]$, the model predictive control (MPC) has attracted attention because of its ability of improving the robustness of the system and having better dynamic control performance in solving the problem of consensus protocol in distributed formation control of multiple mobile robots. Also, MPC is convenient to establish the system model.

To further improve the performance of MPC, researchers investigate MPC combining with robust control $[14,15]$, adaptive control $[16,17]$, and sliding model control [18]. Among them, there are lots of works focusing on overcoming the uncertainty problem of formation control of multiple mobile robots. In the research of multiagent [19], a fault-tolerant controller was designed to compensate the influence of the system uncertainty. By proposing a static nonsmooth event-triggered protocol [20], the influence of the uncertainty is inhibited. Robust and adaptive control algorithms were used [21] for each agent to deal with the system uncertainty associated with leader input and follower agent dynamics. In the study of MAVs [22], adaptive control algorithms were employed locally for each vehicle to solve the uncertainty associated with the system and flying environment. Although these methods mentioned above have disposed of the uncertainty problem of the formation control with MPC, they are not combined with a distributed control. As for the MPC applied in the distributed control (distributed model predictive control (DMPC)), a cooperative DMPC algorithm was reported [23] where the relaxed constraints are used to find the optimization problem including the global optimization objective. This algorithm can be utilized to control the UAV tracking the ground target for real-time path planning [24] and multiple cooperative autonomous ships forming a multiship formation system [25]. To transport the plants and keep the formation at the same time [26], an objective function including trajectory tracking error and formation measurement error was adopted to 
meet the design requirements based on the DMPC. The DMPC is widely used in the research studies above, but the uncertainty is not considered. To the best of our knowledge, although lots of results have been available, the issue of simultaneous system uncertainty and DMPC has not been fully studied to date.

In practical application, the parameters in the model may not be accurate, and it is difficult to get an accurate model of system $[27,28]$. Due to the uncertainty of the model, it is almost impossible to predict the future accurately, and the mismatch factors must be noticed. The model uncertainty of the system is ignored in the DMPC algorithm. In addition, the DMPC is too optimistic in the prediction process which would cause the prediction results deviating from the actual effect and lead to many restrictions on the decoupling of the distributed system [29, 30]. For this reason, it is necessary to study the DMPC with system uncertainty. A robust model predictive control approach [31] was suggested to achieve the path tracking of an omnidirectional robot with system uncertainty. The design of robust distributed MPC controller was casted [32] into solving a linear matrix inequality (LMI) optimization problem. A global performance was achieved by using an iterative algorithm. However, the iterative algorithm would cause the jitter fluctuation of the convergence curve and a low computational efficiency [33,34]. A novel model-free adaptive SMC approach for unknown discrete nonlinear processes was designed [35] by bringing the merits of datadriven control strategy and SMC scheme together, which made the system with model uncertainty of strong robustness. A data-driven hierarchical control (DHC) structure was introduced [36] to ameliorate the performance of systems under the effect of the system uncertainty. The above references demonstrate both robust model predictive control and data-driven strategy which are effective methods to work out the problem of system uncertainty so that it is very important to study the data-driven robust distributed model predictive control with system uncertainty.

In this paper, a data-driven robust distributed model predictive control (data-driven RDMPC) with model uncertainty is proposed for multiple mobile robots. The main control method in this paper has these advantages. (1) The data-driven strategy is applied in this method using the historical data representing the future predictive value so that data processing is not limited to the model and the conservatism of system can be reduced. (2) The robust performance objective not the quadratic performance objective is chosen in this method to solve the optimization problem. As a result, the system can consider the model uncertainty, the fluctuation of the convergence curve can be decreased, and also the efficiency and accuracy of the algorithm can be promoted.

This paper is arranged as follows. The control problem is described in Section 2. In Section 3, a data-driven distributed model predictive controller is designed with considering the system uncertainty, and the stability of the system is analyzed. Section 4 presents a simulation example to illustrate the effectiveness of the method. Section 5 puts forward some conclusions.

\section{Problem Formation}

2.1. Notations and Graph Theory. Throughout the paper, the following notations will be used. The function $X(k+\Delta k \mid k)$ means the predicted value of variables $X(k+\Delta k)$ in time $k$. $X(k \mid k)=X(k)$ represents the variable $x$ in current time and can be measured by sensors. And a quadric form function is given as $x_{Q}^{2}=x^{T} Q x$ where $Q>0$.

This paper examines the mobile robot formation control with the distributed control method. A finite index is set to mark the mobile robot, making each robot node as $1,2, \ldots, n$. A directed and fixed graph $G$ is $(V, \varepsilon, A) . V=\left\{v_{1}, v_{2}, \ldots, v_{n}\right\}$ is a set of nodes, where $v_{i}$ is referred to the $i$-th mobile robot. $\varepsilon \in V \times V$ is the edge set. $\varepsilon_{i j}=\left(v_{i}, v_{j}\right) \in \varepsilon$ indicates that the $i$ th robot can obtain the information from $j$-th robot. $A=$ $\left[a_{i j}\right]$ is the weighted adjacency matrix of G. If $\varepsilon_{i j} \in \varepsilon$, we have $a_{i j}=a_{j i}>0$ while $a_{i j}=0$ and $a_{i i}=0$.

2.2. Mathematical Model. The mobile robot $i$ has $q_{i}=\left[\begin{array}{lll}x_{i} & y_{i} & \alpha_{i}\end{array}\right]^{T}$ on their path $\Gamma_{i}, i=1,2, \ldots, n$. The statement of the robot $j$ communicating with the robot $i$ is $q_{j}=\left[\begin{array}{lll}x_{j} & y_{j} & \alpha_{j}\end{array}\right]^{T}$, and $\left(x_{i}, y_{i}, \alpha_{i}\right)$ and $\left(x_{j}, y_{j}, \alpha_{j}\right)$ are the position $(x, y)$ and orientation $\alpha$ of robot $i$ and $j$, respectively. The desired relative statement of robot $i$ and $j$ is $p_{i j}=\left[\begin{array}{lll}x_{i j}^{r} & y_{i j}^{r} & \alpha_{i j}^{r}\end{array}\right]^{T}$ where $x_{i j}^{r}, y_{i j}^{r}$, and $\alpha_{i j}^{r}$ represent the desired relative position and relative orientation. The kinematic models of $i$ and $j$ are established as follows:

$$
\begin{aligned}
& {\left[\begin{array}{c}
\dot{x}_{i} \\
\dot{y}_{i} \\
\dot{\alpha}_{i}
\end{array}\right]=\left[\begin{array}{cc}
\cos \alpha_{i} & 0 \\
\sin \alpha_{i} & 0 \\
0 & 1
\end{array}\right]\left[\begin{array}{c}
v_{i} \\
\omega_{i}
\end{array}\right],} \\
& {\left[\begin{array}{c}
\dot{x}_{j} \\
\dot{y}_{j} \\
\dot{\alpha}_{j}
\end{array}\right]=\left[\begin{array}{cc}
\cos \alpha_{j} & 0 \\
\sin \alpha_{j} & 0 \\
0 & 1
\end{array}\right]\left[\begin{array}{c}
v_{j} \\
\omega_{j}
\end{array}\right],}
\end{aligned}
$$

where $v_{i}$ and $\omega_{i}$ represent the control input of the forward and angular velocities of robot $i$ and $v_{j}$ and $\omega_{j}$ represent the control input of the forward and angular velocities of robot $j$, and then the relative statement between them can be expressed as follows:

$$
\left[\begin{array}{l}
x_{i j} \\
y_{i j} \\
\alpha_{i j}
\end{array}\right]=\left[\begin{array}{ccc}
\cos \alpha_{i} & \sin \alpha_{i} & 0 \\
-\sin \alpha_{i} & \cos \alpha_{i} & 0 \\
0 & 0 & 1
\end{array}\right]\left(q_{i}-q_{j}-p_{i j}\right)
$$

By taking the derivative of (3) and combining with (1) and (2), the following system is obtained:

$$
\left\{\begin{array}{l}
\dot{x}_{i j}=\omega_{i}\left(y_{i j}-y_{i j}^{r}\right)-v_{i}+v_{j} \cos \left(\alpha_{i j}-\alpha_{i j}^{r}\right), \\
\dot{y}_{i j}=-\omega_{i}\left(x_{i j}-x_{i j}^{r}\right)+v_{j} \sin \left(\alpha_{i j}-\alpha_{i j}^{r}\right), \\
\dot{\alpha}_{i j}=\omega_{i}-\omega_{j}-\omega_{i j}^{r},
\end{array}\right.
$$

where $\omega_{i j}^{r}$ is the desired angular speed error between robot $i$ and robot $j$. System (4) between robot $i$ and robot $j$ can be linearized around $X_{i j}=0, U_{i j}=0$ as follows: 


$$
\dot{X}_{i j}=A_{l i} X_{i j}+B_{l i} U_{i j}
$$

where $A_{l i}=\left[\begin{array}{ccc}0 & \omega_{i} & 0 \\ -\omega_{i} & 0 & v_{j} \\ 0 & 0 & 0\end{array}\right], \quad B_{l i}=\left[\begin{array}{ll}1 & 0 \\ 0 & 0 \\ 0 & 1\end{array}\right], \quad$ and $X_{i j}=$ $\left[\begin{array}{lll}x_{i j}-x_{i j}^{r} & y_{i j}-y_{i j}^{r} & \alpha_{i j}-\alpha_{i j}^{r}\end{array}\right]^{T}$ are the statement and $U_{i j}=$ $\left[-v_{i}+v_{j} \cos \left(\alpha_{i j}-\alpha_{i j}^{r}\right) \omega_{i}-\omega_{j}-\omega_{i j}^{r}\right]^{T}$ is the control input. Considering the communication between robot $i$ and other robots, the linearized system of robot $i$ is written as follows:

$$
\dot{X}_{i}=A_{l i} X_{i}+B_{l i} U_{i}
$$

where $X_{i}=\sum_{s=1}^{m} a_{i j} X_{i j}$ and $U_{i}=\sum_{s=1}^{m} a_{i j} U_{i j}$, and the elements of $X_{i}$ are defined as $X_{i}=\left[\begin{array}{lll}x_{B i} & y_{B i} & \alpha_{B i}\end{array}\right]^{T}$; therefore, $x_{B i}=\sum_{s=1}^{m} a_{i j}\left(x_{i j}-x_{i j}^{r}\right), \quad y_{B i}=\sum_{s=1}^{m} a_{i j}\left(y_{i j}-y_{i j}^{r}\right), \quad$ and $\alpha_{B i}=\sum_{s=1}^{m} a_{i j}\left(\alpha_{i j}-\alpha_{i j}^{r}\right)$. It should be noted that $X_{i}$ is the sum of relative position errors between each mobile robot $i$ and robot $j$ and $m$ is the number of robots communicating with robot $i$. After discretizing equation (6), the normative system of mobile robot $i$ is as follows:

$$
X_{i \text { norm }}(k+1)=A_{0 i} X_{i}(k)+B_{0 i} U_{i}(k),
$$

where $A_{0 i}=e^{A_{l i} T}$ and $B_{0 i}=\int_{0}^{T} e^{A_{l i} \tau} \mathrm{d} \tau B_{l i}, T$ is the sampling period. Because of the uncertainty, the system will produce errors. Besides, the linearization process will also bring the errors to the system. To deal with these two errors concurrently, an uncertainty term $\Delta X_{i}(k)$ related to control input $U_{i}(k)$ and state variable $X_{i}(k)$ is considered to describe the uncertainty of the system as follows:

$$
\Delta X_{i}(k)=\delta_{i}\left(\Delta C_{i} X_{i}(k)+\Delta D_{i} U_{i}(k)\right)
$$

where $\Delta C_{i}$ and $\Delta D_{i}$ are known matrices. $\delta_{i}$ is an unknown but bounded time-independent matrix and satisfies $\delta_{i 2} \equiv \sigma\left(\delta_{i}\right) \leq 1$. Then, considering the external disturbance of the mobile robot, the bounded disturbance term $d_{i}(k) \in R^{3 \times 1}$ is added. The uncertain but bounded system model of mobile robot $i$ is shown as follows:

$$
x_{i}(k+1)=x_{i \text { norm }}(k+1)+\Delta x_{i}(k)+d_{i}(k)=\left(A_{0 i}+\delta_{i} \Delta C_{i}\right) X_{i}(k)+\left(B_{0 i}+\delta_{i} \Delta D_{i}\right) U_{i}(k)+d_{i}(k),
$$

and the parameter of this uncertain system model is $\left[\begin{array}{ll}A_{i} & B_{i}\end{array}\right]=\left[A_{0 i}+\delta_{i} \Delta C_{i} B_{0 i}+\delta_{i} \Delta D_{i}\right] \in \Omega$.

In the control process, the actuator usually bears limited control force. Therefore, it is very necessary to consider the saturation constraints of the input and the hard constraints of the state variables in the controller design [37]. Otherwise, the control will be inaccurate because of the control input and state variables exceed the limits, and this situation will inevitably reduce the tracking performance of the control system [38]. Therefore, saturation constraint (10) to limit the control input and hard constraint (11) to limit the state variable are added as follows:

$$
\begin{gathered}
\left|U_{i}(k)_{c}\right| \leq U_{i}(k)_{c, \text { max }}, \quad c=1,2, \\
\left|F_{i c} X_{i}(k)\right| \leq X_{i}(k)_{c, \text { max }}, \quad c=1,2,3,
\end{gathered}
$$

where $U_{i}(k)_{c, \max }$ and $X_{i}(k)_{c, \text { max }}$ are the maximum of $c$-th element of $U_{i}(k)$ and $X_{i}(k)$ and $F_{i c}$ is the $c$-th row of the assigned matrix $F_{i} \in R^{3 \times 3}$.

\section{Controller Design and Stability Analysis}

3.1. Controller Design. It is worth emphasizing that each mobile robot has its own independent MPC controller. By communicating with each other, these robots receive the information which can form the coupling conditions to solve the optimal control problem online on their respective controllers. For each robot MPC controller, in the absence of an accurate model, the performance of solving the optimization problem which uses the method of quadratic performance objective minimization will be very poor, and the computational load will be large. So, the method applying the robust performance objective minimization to replace the quadratic performance objective minimization to establish the optimization problem is considered for the controller design. A feedback gain $K$ is set to adjust $U(k)$ and is continuously updated by the optimization problem. The optimization problem is shown as follows:

$$
\begin{aligned}
& \min _{U_{i}} \max _{\left[\begin{array}{ll}
A_{i} & B_{i}
\end{array}\right] \in \Omega} J_{i 0}^{\infty}(k) \\
& \text { s.t. (6) }-(10) .
\end{aligned}
$$

In order to propose an appropriate objective function, a trajectory parameter is needed to be included in the objective function of the normative system to construct the coupling relationship. A parameter $\zeta_{i}$ is defined to describe the trajectory $\Gamma_{i}\left(\zeta_{i}\right)=\left[\begin{array}{lll}x_{\Gamma i}\left(\zeta_{i}\right) & y_{\Gamma i}\left(\zeta_{i}\right) & \alpha_{\Gamma i}\left(\zeta_{i}\right)\end{array}\right]$ of mobile robot $i$, which is an iterative function related to control input, forward, and angular velocities:

$$
\zeta_{i}(k+1)=\zeta_{i}(k)+M_{i} U_{i}(k)+N_{i}\left[\begin{array}{ll}
v_{i} & \omega_{i}
\end{array}\right]^{T},
$$

where $M_{i}$ and $N_{i}$ are the given parameter matrix. Defining $\zeta_{i j}^{r}$ as the desired value of the trajectory parameter difference between robot $i$ and robot $j$, it is an important parameter to predict the trajectory of robot $j$ through robot $i$ and then the trajectory of robot $j$ can be described as $\Gamma_{j}\left(\zeta_{i}\right)=\left[x_{\Gamma i}\right.$ $\left.\left(\zeta_{i}\right)+x_{\Gamma i j}\left(\zeta_{i j}^{r}\right) y_{\Gamma i}\left(\zeta_{i}\right)+y_{\Gamma i j}\left(\zeta_{i j}^{r}\right) \alpha_{\Gamma i}\left(\zeta_{i}\right)+\alpha_{\Gamma i j}\left(\zeta_{i j}^{r}\right)\right]$. The information which robots interact includes their position states and trajectory parameters. The position states and the trajectory parameters are used to calculate $X_{i j}$ and form coupling conditions, respectively. The objective function of the normative system coupled with $\zeta_{j}$ is as follows: 


$$
\begin{aligned}
J_{i 0}^{\infty}(k) & =\sum_{\Delta k=0}^{\infty}\left\|X_{i}(k+\Delta k+1 \mid k)\right\|_{\bar{Q}_{i}}^{2}+\left\|U_{i}(k+\Delta k \mid k)\right\|_{\bar{R}_{i}}^{2}+\sum_{s=1}^{m}\left\|\zeta_{i}(k+\Delta k \mid k)-\zeta_{j}(k+\Delta k \mid k)-\zeta_{i j}^{r}\right\|_{\bar{W}_{i, l}}^{2} \\
& =\sum_{\Delta k=0}^{\infty}\left\|A_{i} X_{i}(k+\Delta k \mid k)+B_{i} U_{i}(k+\Delta k \mid k)\right\|_{\bar{Q}_{i}}^{2}+\left\|U_{i}(k+\Delta k \mid k)\right\|_{\bar{R}_{i}}^{2}+\sum_{s=1}^{m}\left\|H_{i} U_{i}(k+\Delta k \mid k)-\psi_{i j}\right\|_{\bar{W}_{i j}}^{2},
\end{aligned}
$$

where $\psi_{i j}=\Theta_{j}(k+\Delta k \mid k)+H_{i}\left(U_{j}(k+\Delta k \mid k)+\bar{v}_{j}\right)+\Theta_{i j}^{r}-$ $\Theta_{i}(k+\Delta k \mid k)-H_{i} \bar{v}_{i}, \bar{Q}_{i}=\operatorname{diag}\left\{Q_{i}, Q_{i}, Q_{i}\right\}, Q_{i}>0, \bar{R}_{i}=\operatorname{diag}$ $\left\{R_{i}, R_{i}\right\}, \quad R_{i}>0, \quad \bar{W}_{i j}=\operatorname{diag}\left\{w_{i j}, w_{i j}, w_{i j}\right\}, \quad w_{i j}>0, \quad \Theta_{i}=$ $\left[\zeta_{i}, \zeta_{i}, \zeta_{i}\right]^{T}, \quad \Theta_{j}=\left[\zeta_{j}, \zeta_{j}, \zeta_{j}\right]^{T}, \quad \Theta_{i j}^{r}=\left[\zeta_{i j}^{r}, \zeta_{i j}^{r}, \zeta_{i j}^{r}\right]^{T}, \quad \bar{v}_{i}=$ $\left[\begin{array}{ll}v_{i} & \omega_{i}\end{array}\right]^{T}, \bar{v}_{j}=\left[\begin{array}{ll}v_{j} & \omega_{j}\end{array}\right]^{T}$, and $H_{i}=\left[\begin{array}{lll}M_{i} & M_{i} & M_{i}\end{array}\right]^{T}$.

For the uncertain system (9), compared with $X_{i}(k \mid k)$, we focus on $X_{i}(k+1 \mid k)$ to control the system and get $X_{i}(k+$ $1 \mid k)$ from the historical data without uncertain factors. $X_{i}(k+1 \mid k)$ should be limited to the feasible region $Z=$ $\left\{z \mid z^{T} \widetilde{Q}_{i}^{-1} z \leq 1\right\}$ of the optimization problem, so there is $X_{i}^{T}(k+1 \mid k) \widetilde{Q}_{i}^{-1} X_{i}(k+1 \mid k) \leq 1$, and using the Schur complement, we can get the following:

$$
\left[\begin{array}{cc}
1 & * \\
X_{i}(k+1 \mid k) & \widetilde{Q}_{i}
\end{array}\right] \geq 0 .
$$

If the ellipsoid $Z$ is an invariant set, then the objective function can be divided into two parts as follows:

$$
\begin{aligned}
J_{i 0}^{\infty}(k)= & \left\|A_{0 i} X_{i}(k \mid k)+B_{0 i} U_{i}(k \mid k)\right\|_{\bar{Q}_{i}}^{2}+\left\|U_{i}(k \mid k)\right\|_{\bar{R}_{i}}^{2}+\sum_{s=1}^{m}\left\|H_{i} U_{i}(k \mid k)-\psi_{i j}\right\|_{\bar{W}_{i j}}^{2} \\
& +\sum_{\Delta k=1}^{\infty}\left\|A_{0 i} X_{i}(k+\Delta k \mid k)+B_{0 i} U_{i}(k+\Delta k \mid k)\right\|_{\bar{Q}_{i}}^{2}+\left\|U_{i}(k+\Delta k \mid k)\right\|_{\bar{R}_{i}}^{2}+\sum_{s=1}^{m}\left\|H_{i} U_{i}(k+\Delta k \mid k) \psi_{i j}\right\|_{\bar{W}_{i j}}^{2}=J_{i 0}(k)+J_{i 1}^{\infty}(k) .
\end{aligned}
$$

The optimization problem is transformed into solving a set of state feedback laws $U_{i}=K_{i} X_{i}$. In the case, the controller objective minimizes the upper bound of the worstcase objective function. Let $\gamma_{1 i}$ and $\gamma_{2 i}$ be the upper bound of objective function $J_{i 0}(k)$ and $J_{i 1}^{\infty}(k)$, respectively.

Theorem 1. For the uncertain system (9), if there exists $\Lambda_{1}=\operatorname{diag}\left\{\lambda_{1}, \lambda_{2}, \lambda_{3}\right\}>0$, then the system will be stable and $Z$ is an invariant set when following conditions are satisfied:

$$
\left[\begin{array}{ccccc}
\widetilde{Q}_{i} & * & * & * & * \\
A_{0 i} \widetilde{Q}_{i}+B_{0 i} Y_{i} & \widetilde{Q}_{i} & * & * & * \\
\Delta C_{i} \widetilde{Q}_{i}+\Delta D_{i} Y_{i} & 0 & \Lambda_{1} & * & * \\
\left(A_{0 i}^{T} \bar{Q}_{i} A_{0 i}\right)^{0.5} \widetilde{Q}_{i} & 0 & 0 & \gamma_{2 i} I & * \\
S_{i}^{0.5} Y_{i} & 0 & 0 & 0 & \gamma_{2 i} I
\end{array}\right] \geq 0,
$$

$$
\begin{gathered}
{\left[\begin{array}{cc}
G_{i} & * \\
Y_{i} & \widetilde{Q}_{i}
\end{array}\right] \geq 0,} \\
G_{i c c} \leq U_{i c}^{2}, \quad c=1,2, \\
{\left[\begin{array}{cc}
L_{i} & * \\
\widetilde{Q}_{i} F_{i}^{T} & \widetilde{Q}_{i}
\end{array}\right] \geq 0,} \\
L_{i c c} \leq X_{i c}^{2}, \quad c=1,2,3,
\end{gathered}
$$

where $S=B_{0 i}^{T} \bar{Q}_{i} B_{0 i}+\bar{R}_{i}+\sum_{s=1}^{m} H_{i}^{T} \bar{W}_{i j} H_{i}, G_{i c c}$ and $L_{i c c}$ represent the $c$-th row and $c$-th column of the matrix, and $U_{i c}$ and $X_{i c}$ represent the $c$-th element of the control input and state variable.

Proof of Theorem 1. If $X_{i}(k+1 \mid k)$ is restricted in the ellipsoid $Z$ and $Z$ is an invariant set, then the objective function in infinite time domain can be regarded as two parts of $(16)$, where $J_{i 0}^{1}(k)$ is finite time domain. And the stability of the system can be analyzed by aiming at $J_{i 1}^{\infty}(k)$ which is in infinite time domain. Consider a Lyapunov quadratic function $V\left(X_{i}\right)=X_{i}^{T} P_{i} X_{i}, P_{i}>0$. For any time, $A_{i}$ and $B_{i}$ satisfy $\left[\begin{array}{ll}A_{i} & B_{i}\end{array}\right] \in \Omega$. According to the Lyapunov 
stability criterion, $V\left(X_{i}\right)$ should meet the following inequality:

$$
\begin{aligned}
V\left(X_{i}(k+j+1 \mid k)\right)-V\left(X_{i}(k+j \mid k)\right) \leq & {\left[\left\|A_{0 i} X_{i}(k+\Delta k \mid k)+B_{0 i} U_{i}(k+\Delta k \mid k)\right\|_{\bar{Q}_{i}}^{2}+\left\|U_{i}(k+\Delta k \mid k)\right\|_{\bar{R}_{i}}^{2}\right.} \\
& \left.+\sum_{s=1}^{m}\left\|H_{i} U_{i}(k+j \mid k)-\psi_{i, l}\right\|_{\bar{W}_{i, l}}^{2}\right]
\end{aligned}
$$

Summing (21) from $i=1$ to $i=\infty$ and requiring $X_{i}(\infty \mid k)=0$ or $V\left(X_{i}(\infty \mid k)\right)=0$, the result is as follows:

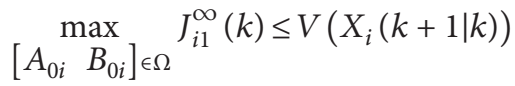

$$
\begin{aligned}
& =X_{i}^{T}(k+1 \mid k) P_{i} X_{i}(k+1 \mid k) \leq \gamma_{2 i} \text {. }
\end{aligned}
$$

There is $X_{i}^{T}(k+1 \mid k) \widetilde{Q}_{i}^{-1} X_{i}(k+1 \mid k) \leq 1$, then let $P_{i}=\gamma_{2 i} \widetilde{Q}_{i}^{-1}$. Substituting (9) and state feedback law $U_{i}=$ $K_{i} X_{i}$ into (21), we can get the following:

$$
\begin{aligned}
& X_{i}^{T}(k+\Delta k \mid k)\left\{\left(A_{i}+B_{i} K_{i}\right)^{T} P_{i}\left(A_{i}+B_{i} K_{i}\right)-P_{i}+\left(G_{i}+B_{i} K_{i}\right)^{T} \bar{Q}_{i}\left(G_{i}+B_{i} K_{i}\right)+K_{i}^{T} \bar{R}_{i} K_{i}+\sum_{s=1}^{m}\left(H_{i} K_{i}\right)^{T} \bar{W}_{i j}\left(H_{i} K_{i}\right)\right\} \\
& X_{i}(k+\Delta k \mid k)+d_{i}^{T}(k) P_{i} d_{i}(k)-\sum_{s=1}^{m} \psi_{i j}^{T} \bar{W}_{i j} \psi_{i j} \leq 0,
\end{aligned}
$$

where (23) can be regarded as two parts, and they are as follows:

$$
\begin{array}{r}
\left(A_{i}+B_{i} K_{i}\right)^{T} P_{i}\left(A_{i}+B_{i} K_{i}\right)-P_{i}+\left(G_{i}+B_{i} K_{i}\right)^{T} \bar{Q}_{i}\left(G_{i}+B_{i} K_{i}\right)+K_{i}^{T} \bar{R}_{i} K_{i}+\sum_{s=1}^{m}\left(H_{i} K_{i}\right)^{T} \bar{W}_{i j}\left(H_{i} K_{i}\right) \leq 0 \\
d_{i}^{T}(k) P_{i} d_{i}(k)-\sum_{s=1}^{m} \psi_{i j}^{T} \bar{W}_{i j} \psi_{i j} \leq 0 .
\end{array}
$$

In the above formula, the feedback gain is defined as $K_{i}=Y_{i} \widetilde{Q}_{i}^{-1}$, and the solution of $Y_{i}$ is given by the optimization problem. Substituting $\left[\begin{array}{ll}A_{i} & B_{i}\end{array}\right]=\left[A_{0 i}+\delta_{i}\right.$ $\left.\Delta C_{i} B_{0 i}+\delta_{i} \Delta D_{i}\right]$, feedback gain $K_{i}=Y_{i} \widetilde{Q}_{i}^{-1}$ and $P_{i}=\gamma_{2 i} \widetilde{Q}_{i}^{-1}$ into (23a) and (23b), and transforming (23a) and (23b) into matrix inequality form, the results are (19) and (20), respectively. Systems (19) and (20) guarantee the positive invariance of $Z$. In the invariant set $Z$, the saturation constraint (10) and hard constraint (11) are expressed as (19) and (20) in matrix form, respectively, and the control input is limited by $G_{i}$, and the state variable is limited by $L_{i}$.

Remark 1. In Theorem 1, equation (17) can get the constraint that the upper bound $\gamma_{2 i}$ of $J_{i 1}^{\infty}(k)$ is satisfied when the system is stable. After the upper bound $\gamma_{1 i}$ of $J_{i 0}(k)$ can also be expressed as some concrete constraints, the problem of minimizing the upper bound of the objective function in the worst case can be formulated and solved. And with the update of feedback gain, $X_{i}(k)$ is always limited in the ellipsoid $Z$ at any time in the optimization process.

Theorem 2. The upper bound $\gamma_{1 i}$ of $J_{i 0}(k)$ satisfies the following relation:

$$
\left[\begin{array}{cc}
\gamma_{1 i}^{*} & * \\
U_{i}(k \mid k) & S_{i}
\end{array}\right] \geq 0,
$$

where $\psi_{i j}+\gamma_{1 i}^{*}$

$$
\gamma_{1 i}=x_{i}^{T}(k \mid k)\left(A_{0 i}^{T} \bar{Q}_{i} A_{0 i}\right) x_{i}(k \mid k)-\sum_{s=1}^{m} \psi_{i j}^{T} \bar{W}_{i j}
$$

Proof of Theorem 2. The objective function of $J_{i 0}(k)$ is as follows: 


$$
\begin{aligned}
J_{i 0}(k) & =\left\|A_{0 i} X_{i}(k \mid k)+B_{0 i} U_{i}(k \mid k)\right\|_{\bar{Q}_{i}}^{2}+\left\|U_{i}(k \mid k)\right\|_{\bar{R}_{i}}^{2}+\sum_{s=1}^{m}\left\|H_{i} u_{i}(k \mid k)-\psi_{i j}\right\|_{\bar{W}_{i j}}^{2} \\
& =x_{i}^{T}(k \mid k)\left(A_{0 i}^{T} \bar{Q}_{i} A_{0 i}\right) x_{i}(k \mid k)+u_{i}^{T} S_{i} u_{i}-\sum_{s=1}^{m} \psi_{i j}^{T} \bar{W}_{i j} \psi_{i j} \leq \gamma_{1 i},
\end{aligned}
$$

where $x_{i}(k \mid k)$ can be measured by sensors and exists in historical data. $\psi_{i j}$ is the function related to $\zeta_{i}, \zeta_{j}$, and $u_{j} . \zeta_{i}$ can also be measured by sensors, and $\zeta_{j}$ and $u_{j}$ can interact with mobile robot $j$ through wireless network or Bluetooth technology. Let $u_{i}^{T} S_{i} u_{i} \leq \gamma_{1 i}^{*}$, equation (24) is obtained after Schur complement.

Theorem 2 gives the condition of upper bound $\gamma_{1 i}$ of $J_{i 0}(k)$; then, the upper bound of the objective function in the infinite time in the worst case can be expressed as follows:

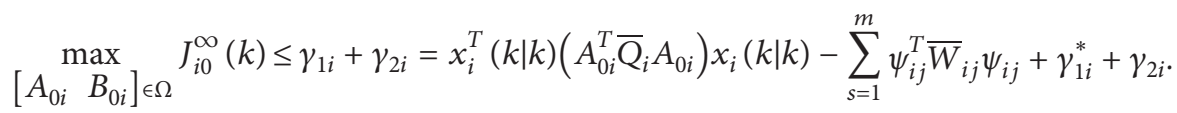

The conditions about the upper bound of the objective function, feedback gain, and other parameters are given in the above theorems with the stable system, which can be used as the constraints of the optimization problem. Then, the optimization problem (12) can be described as LMI problem as follows:

$$
\begin{aligned}
& \min _{\widetilde{Q}_{i}, Y_{i}, G_{i}, L_{i}, \Lambda_{1}, \gamma_{1 i}, \gamma_{2 i}} \gamma_{1 i}^{*}+\gamma_{2 i} \\
& \text { s.t. (15), (17), (18), (19), (20), (24). }
\end{aligned}
$$

As mentioned in the paper above, we focus on $X_{i}(k+$ $1 \mid k)$ not $X_{i}(k \mid k)$ to control the system when solving the optimization problem. At this time, the difficulty is how to use the historical data from the previous time to represent the predicted value of the future time. So, consider the following linear law of historical data:

$$
\begin{aligned}
& X_{i}(k \mid k)=\mathscr{E}_{i k}(1) \widetilde{\delta}_{i 2}(1)+\mathscr{E}_{i k}(2) \widetilde{\delta}_{i 2}(2)+\Delta X_{i}(k), \\
& U_{i}(k \mid k)=\mathscr{E}_{i k}(1) \mho_{i 3}(1)+\mathscr{E}_{i k}(2) \mho_{i 3}(2)+\Delta U_{i}(k),
\end{aligned}
$$

where $\mathscr{E}_{i k} \in R^{1 \times 2}$ represents the coefficient matrix when the historical data are employed to indicate the future forecast value linearly. $\sigma_{i 2}=\left[\begin{array}{lll}X_{i}(k-1 \mid k) & X_{i}(k-2 \mid k)\end{array}\right]^{T}$ and $\sigma_{i 3}=$ $\left[U_{i}(k-1 \mid k) U_{i}(k-2 \mid k)\right]^{T}$ denote storage units containing two sets of historical data of $X_{i}(k \mid k)$ and $U_{i}(k \mid k)$, respectively. When $k=0, X_{i}(k-2 \mid k)=0$ and $U_{i}(k-2 \mid k)=0$. $\Delta X_{i}(k)$ and $\Delta U_{i}(k)$ are the remainders of the errors. Define $\delta_{i 4}=\left[\begin{array}{lll}X_{i}(k \mid k) & X_{i}(k-1 \mid k)\end{array}\right]^{T}$ as the storage unit including two sets of historical data of $X_{i}(k+1 \mid k)$, but this unit has no direct connection with $\widetilde{O}_{i 2}$ and $\widetilde{\sigma}_{i 3}$, and the date of them exists independently. The system does not take the bounded external interference into consideration; after substituting (28) and (29) into the system model, the result is as follows:

$$
X_{i}(k+1 \mid k)=\mathscr{E}_{i k}(1) \widetilde{\delta}_{i 4}(1,:)+\mathscr{E}_{i k}(2) \mho_{i 4}(2,:)+A_{0 i} \Delta X_{i}(k)+B_{0 i} \Delta U_{i}(k)+\delta_{i}\left(\Delta C_{i} X(k)+\Delta D_{i} \Delta U_{i}(k)\right)
$$

Substituting (30) into (15), we can get

$$
\left[\begin{array}{cc}
\mathscr{E}_{i k}(1) \mho_{i 4}(1,:)+\mathscr{E}_{i k}(2) \mho_{i 4}(2,:)+A_{0 i} \Delta X_{i}(k) & * \\
+B_{0 i} \Delta U_{i}(k)+\delta_{i}\left(\Delta C_{i} X(k)+\Delta D_{i} \Delta U_{i}(k)\right) & \widetilde{Q}_{i}
\end{array}\right] \geq 0
$$

After expanding (31), set $\Lambda_{2}=\operatorname{diag}\left\{\lambda_{4}, \lambda_{5}, \lambda_{6}\right\}>0$, and the result is shown as follows:

$$
\left[\begin{array}{ccc}
1 & * & * \\
\mathscr{E}_{i k}(1) \delta_{i 4}(1,:)+\mathscr{E}_{i k}(2) \delta_{i 4}(2,:)+A_{0 i} \Delta X_{i}(k)+B_{0 i} \Delta U_{i}(k) & \widetilde{Q}_{i} & 0 \\
\Delta C_{i} \Delta X_{i}(k)+\Delta D_{i} \Delta U_{i}(k) & 0 & \Lambda_{2}
\end{array}\right] \geq 0
$$

Substituting (29) into (24), we can get 


$$
\left[\begin{array}{cc}
\gamma_{1 i}^{*} & * \\
\mathscr{E}_{i k}(1) \mho_{i 3}(1,:)+\mathscr{E}_{i k}(2) \widetilde{\delta}_{i 3}(2,:)+\Delta U_{i}(k) & S_{i}
\end{array}\right] \geq 0 .
$$

Inequalities (32) and (33) are obtained from historical data, and then the data-driven optimization problem (27) can be expressed as follows:

$$
\begin{aligned}
& \min _{\widetilde{Q}_{i}, Y_{i}, G_{i}, L_{i} \Lambda_{1} \Lambda_{1}, E_{i k}, \Delta X_{i}(k), \Delta U_{i}(k), \gamma_{1 i}^{\prime} \gamma_{2 i}} \\
& \text { s.t (17), (18), (19), (20), (32), (33). }
\end{aligned}
$$

\subsection{Stability Analysis}

Lemma 1. When the system is inspired, the stored energy decreases with time and finally reaches a minimum, and then the system is gradually stable. If there is a positive definite scalar function $V\left(X_{i}\right)$ with negative definite $\dot{V}\left(X_{i}\right)$, the system is stable.

A positive definite Lyapunov function $V\left(X_{i}\right)=X_{i}^{T} P_{i} X_{i}$ is defined in 3.1, where $P_{i}>0$. If $\dot{V}\left(X_{i}\right)$ is negative definite, the system is asymptotically stable. The condition for $\dot{V}\left(X_{i}\right)$ to be negative definite is given in equation (21). By using the robust theory, the analysis of $X_{i}$ is transformed into the analysis of state feedback law $U_{i}=K_{i} X_{i}$, and the conditions (17) and (18) for state feedback gain $K_{i}$ are derived. According to Lemma 1, when (17) and (18) are held, the system will tend to stable.

\section{Results}

In this part, two simulations with a group of mobile robots are conducted to verify the proposed approach.

4.1. Simulation 1. In this simulation, the lane-change trajectory is considered. The desired trajectory is considered as follows:

$$
\Gamma=\left\{\begin{array}{l}
x_{\Gamma}^{r}=-\zeta, \\
y_{\Gamma}^{r}=-\frac{2}{1+e^{-2(\zeta-2)}} .
\end{array}\right.
$$

Let $\quad \zeta_{12}^{r}=-10, \zeta_{23}^{r}=-10, \zeta_{34}^{r}=15, \zeta_{45}^{r}=-10$, and $\zeta_{51}^{r}=15$. The trajectory error of every two mobile robots can be expressed as $x_{\Gamma i j}\left(\zeta_{i j}^{r}\right)=-\zeta_{i j}^{r}$ and $y_{\Gamma i j}=2$. The communication topology is shown in Figure 1. The parameters of robots are given as follows: $Q_{1}=100, S_{1}=15, w_{1, l}=4$, $Q_{2}=100, S_{2}=6, w_{2, l}=4, Q_{3}=120, S_{3}=9.6, w_{3, l}=4$, $Q_{4}=100, S_{4}=6, w_{4, l}=4, Q_{5}=120, S_{5}=9.6$, and $w_{5, l}=4$. Set the sampling period $T=0.1 \mathrm{~s}$ and coefficients $\Delta C_{i}=0, \Delta C_{i}=B_{l i}$, and $M_{i}=N_{i}=\left[\begin{array}{ll}-0.2 & 0.1\end{array}\right]$. Each robot's angle speed and line speed are $\omega=1$ and $v=1$, respectively. Taking the uncertainty set as $\delta_{i}=\operatorname{diag}\{-1,-1,-1\}$, the simulation is carried out for $50 \mathrm{~s}$. The initial state values of each mobile robot are as follows:

$$
\begin{aligned}
& X_{1}(0)=\left[\begin{array}{lll}
0.25 & -0.05 & 0.1
\end{array}\right]^{T}, \zeta_{1}(0)=10, \\
& X_{2}(0)=\left[\begin{array}{lll}
0.1 & 0.03 & 0.15
\end{array}\right]^{T}, \zeta_{2}(0)=20, \\
& X_{3}(0)=\left[\begin{array}{lll}
0.15 & -0.02 & 0.1
\end{array}\right]^{T}, \zeta_{3}(0)=30, \\
& X_{4}(0)=\left[\begin{array}{lll}
0.15 & -0.02 & 0.1
\end{array}\right]^{T}, \zeta_{4}(0)=15, \\
& X_{5}(0)=\left[\begin{array}{lll}
0.15 & -0.02 & 0.1
\end{array}\right]^{T}, \zeta_{5}(0)=25 .
\end{aligned}
$$

The bounded external disturbance of each mobile robot is as follows:

$$
\begin{aligned}
& d_{1}(k)=\left[\begin{array}{l}
\left(\frac{0.2}{2 k+1}+\frac{2}{1000}\right) \cos (0.1 k) \\
\left(\frac{0.2}{k+1}+\frac{2}{1000}\right) \sin (0.1 k) \\
\left(\frac{0.1}{k+1}+\frac{1}{1000}\right) \cos (0.2 k)
\end{array}\right], \\
& d_{2}(k)=\left[\begin{array}{l}
\left(\frac{0.1}{2 k+1}+\frac{1}{1000}\right) \cos (0.1 k) \\
\left(\frac{0.2}{k+1}+\frac{2}{1000}\right) \sin (0.1 k) \\
\left(\frac{0.1}{2 k+1}+\frac{1}{1000}\right) \cos (0.2 k)
\end{array}\right],
\end{aligned}
$$

$$
d_{3}(k)=\left[\begin{array}{c}
\left(\frac{0.1}{2 k+1}+\frac{1}{1000}\right) \cos (0.1 k) \\
\left(\frac{0.15}{k+1}+\frac{1}{1000}\right) \sin (0.1 k) \\
\left(\frac{0.2}{k+1}+\frac{2}{1000}\right) \cos (0.2 k)
\end{array}\right],
$$

$$
\begin{aligned}
& d_{4}(k)=\left[\begin{array}{l}
\left(\frac{0.2}{2 k+1}+\frac{1}{1000}\right) \cos (0.1 k) \\
\left(\frac{0.15}{k+1}+\frac{2}{1000}\right) \sin (0.1 k) \\
\left(\frac{0.1}{k+1}+\frac{1}{1000}\right) \cos (0.2 k)
\end{array}\right], \\
& d_{5}(k)=\left[\begin{array}{l}
\left(\frac{0.1}{2 k+1}+\frac{1}{1000}\right) \cos (0.1 k) \\
\left(\frac{0.2}{k+1}+\frac{2}{1000}\right) \sin (0.1 k) \\
\left(\frac{0.1}{k+1}+\frac{2}{1000}\right) \cos (0.2 k)
\end{array}\right] .
\end{aligned}
$$




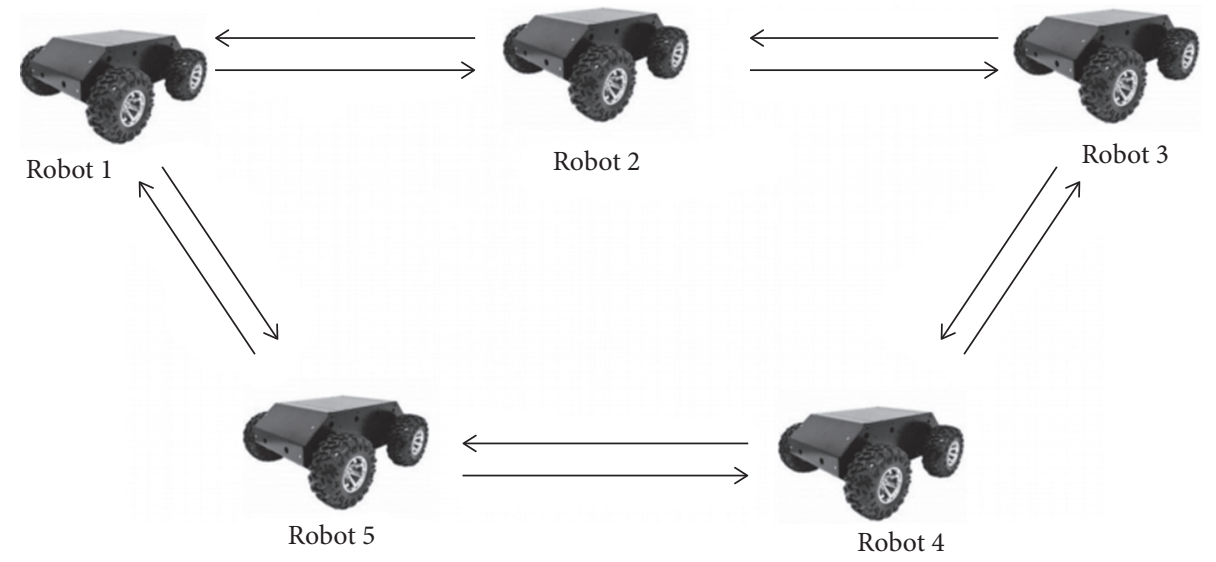

Figure 1: Communication topology: (a) Robot 1; (b) Robot 2; (c) Robot 3; (d) Robot 4; (e) Robot 5.

The simulation is compared with the traditional DMPC. Figure 2 shows the state error $X_{i}$ of each mobile robot. Figure 3 illustrates the absolute value of state error $X_{i}$ of each mobile robot. Figure 4 displays $\zeta_{i j}$ and $\zeta_{i j}^{r}$ of each mobile robot. Figure 5 presents the different values between $\zeta_{i j}$ and $\zeta_{i j}^{r}$ of each mobile robot. Figure 6 demonstrates the trajectories of five mobile robots.

The result in Figure 2 shows that compared with the traditional DMPC, the optimization process of data-driven RDMPC uses the optimal solution instead of the iterative algorithm, which is more efficient and greatly reduces the fluctuation of the convergence curve. In Figure 3, the simulation results with the control target $X_{i}=\left[\begin{array}{lll}0 & 0 & 0\end{array}\right]^{T}$ indicate that the simulation results of data-driven RDMPC are always smaller than those of the traditional DMPC in the process of gradual stabilization, which highlights the more accurate results of the data-driven RDMPC algorithm. From Figure 4 , it is obvious that $\zeta_{i j}$ and $\zeta_{i j}^{r}$ almost coincide. To see errors, although the simulation results fluctuate, the different values representing $\zeta_{i j}-\zeta_{i j}^{r}$ of each mobile robot are always about $10^{-13}$ as shown in Figure 5, and such a small error indicates that the parameter differences among mobile robots converge to the expected value $\zeta_{i j}^{r}$ between them. Figure 6 displays the trajectories of five robots, where the triangle, circle, and diamond represent the initial position, position at $30 \mathrm{~s}$, and the position at $50 \mathrm{~s}$ of each mobile robot, respectively. It can be seen that five mobile robots start from their respective starting points and keep their formation shape in the process of advancing according to each ideal trajectory.

4.2. Simulation 2. In this simulation, we choose the sine function as the trajectory. The desired trajectory is considered as follows:

$$
\Gamma=\left\{\begin{array}{l}
x_{\Gamma}^{r}=\zeta, \\
y_{\Gamma}^{r}=\sin (0.2 \zeta) .
\end{array}\right.
$$

Let $\zeta_{12}^{r}=-1, \zeta_{23}^{r}=-1, \zeta_{34}^{r}=-1, \zeta_{45}^{r}=-1$, and $\zeta_{51}^{r}=4$. The trajectory error between every two mobile robots can be expressed as $x_{\Gamma i j}\left(\zeta_{i j}^{r}\right)=-\zeta_{i j}^{r}$ and $y_{\Gamma i j}=3$. The communication topology is shown in Figure 1. The parameters and the bounded external disturbances of five mobile robots are the same as simulation 1 . Taking the uncertainty set as $\delta_{i}=\operatorname{diag}\{-0.5,-0.5,-0.5\}$, the simulation is carried out for $50 \mathrm{~s}$. The initial state values of each mobile robot are follows:

$$
\begin{aligned}
& X_{1}(0)=\left[\begin{array}{lll}
1 & 1 & 1
\end{array}\right]^{T}, \zeta_{1}(0)=1, \\
& X_{2}(0)=\left[\begin{array}{lll}
1 & -2 & 1
\end{array}\right]^{T}, \zeta_{2}(0)=2, \\
& X_{3}(0)=\left[\begin{array}{lll}
-1.5 & 2 & 1
\end{array}\right]^{T}, \zeta_{3}(0)=3, \\
& X_{4}(0)=\left[\begin{array}{lll}
1.5 & -2 & 1
\end{array}\right]^{T}, \zeta_{4}(0)=4, \\
& X_{5}(0)=\left[\begin{array}{lll}
1 & 2 & -1.5
\end{array}\right]^{T}, \zeta_{5}(0)=5 .
\end{aligned}
$$

Comparing with the traditional DMPC, Figure 7 reveals the state error $X_{i}$ of each mobile robot. Figure 8 shows the absolute value of state error $X_{i}$ of each mobile robot. The trajectory parameter difference $\zeta_{i j}$ and the desired trajectory parameter difference $\zeta_{i j}^{r}$ of each mobile robot are shown in Figure 9. Figure 10 indicates the different values between $\zeta_{i j}$ and $\zeta_{i j}^{r}$ of each mobile robot. Figure 11 displays the trajectories of five robots.

Through the simulation result, we can see that the fluctuation of the convergence curve using the data-driven RDMPC method is obviously been reduced than that using the traditional DMPC method in Figure 7. Taking the absolute value of $X_{i}$, the result of data-driven RDMPC is more accurate than that of traditional DMPC as indicated in Figure 8 . The simulation result of path parameter differences $\zeta_{i j}$ of each mobile robot shown in Figure 9 is very close to $\zeta_{i j}^{r}$ after analyzing the different values between them of each mobile robot in Figure 10. And the difference values are always about $10^{-14}$ or $10^{-15}$, and we can think that $\zeta_{i j}$ tracks $\zeta_{i j}^{r}$ well. Figure 11 displays the trajectories of five robots, where the triangle, circle, and diamond represent the initial position, position at $30 \mathrm{~s}$, and the position at $50 \mathrm{~s}$ of each mobile robot, respectively; these five mobile robots keep their formation shapes ideally. 

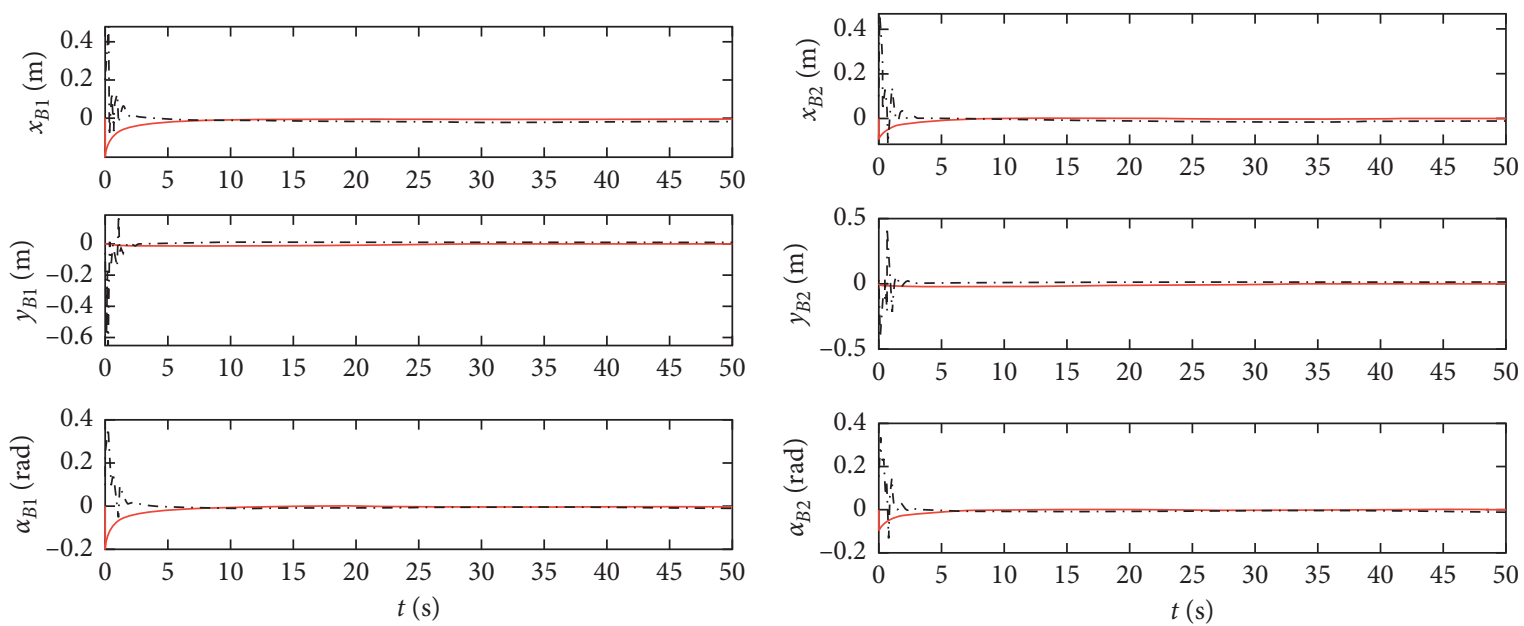

— Data-driven RDMPC

... DMPC

— Data-driven RDMPC

-. - DMPC

(a)

(b)
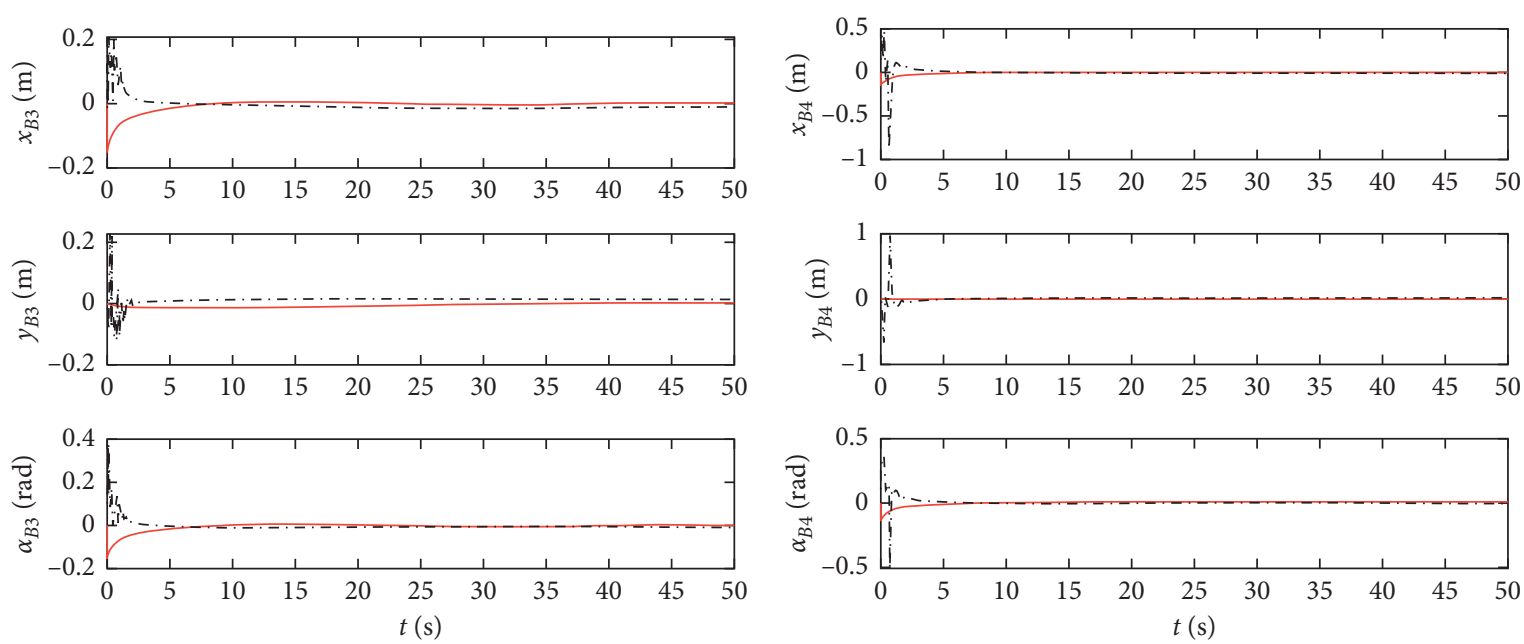

— Data-driven RDMPC

-.. DMPC

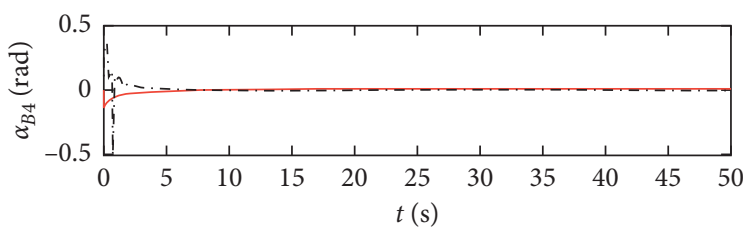

— Data-driven RDMPC

-. - DMPC

(c)

(d)
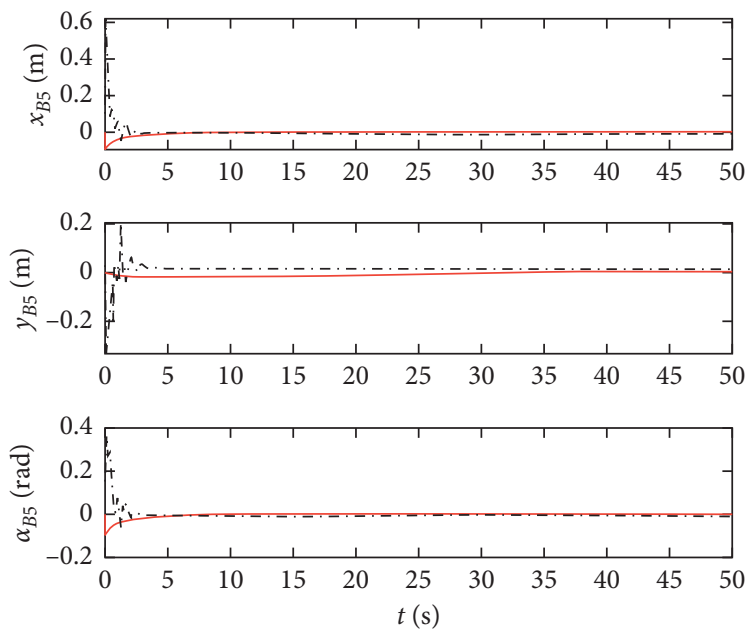

— Data-driven RDMPC

... DMPC

(e)

Figure 2: The state error $X_{i}$ of each mobile robot: (a) Robot 1; (b) Robot 2; (c) Robot 3; (d) Robot 4; (e) Robot 5. 

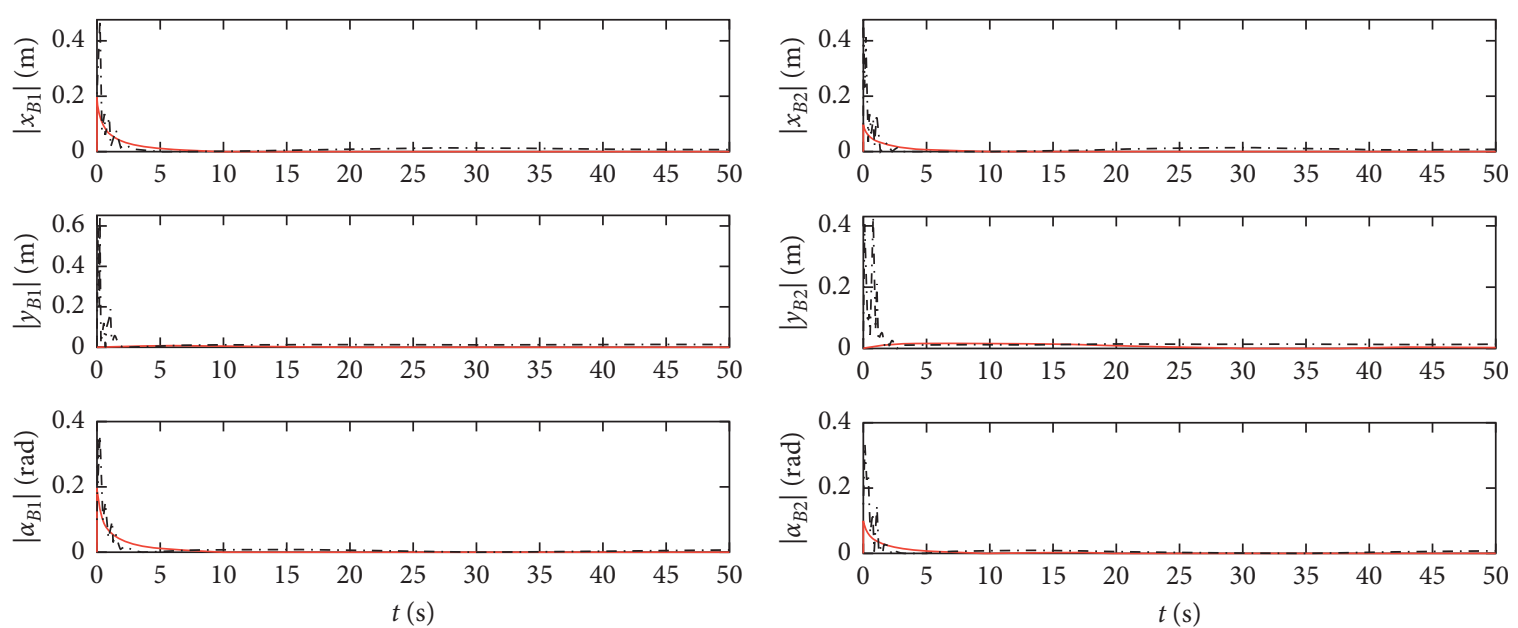

Data-driven RDMPC

-.. DMPC

— Data-driven RDMPC

-. DMPC

(a)

(b)
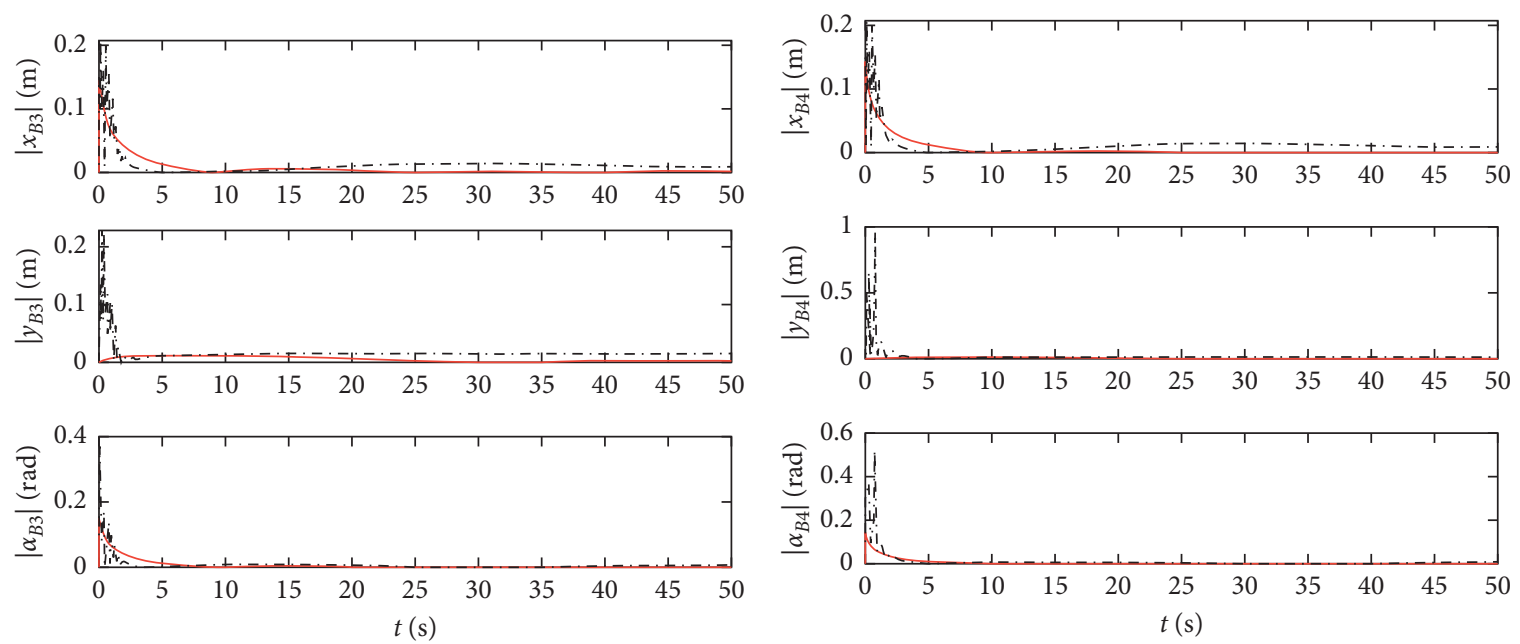

_ Data-driven RDMPC

- Data-driven RDMPC

...- DMPC

-.- DMPC

(c)

(d)
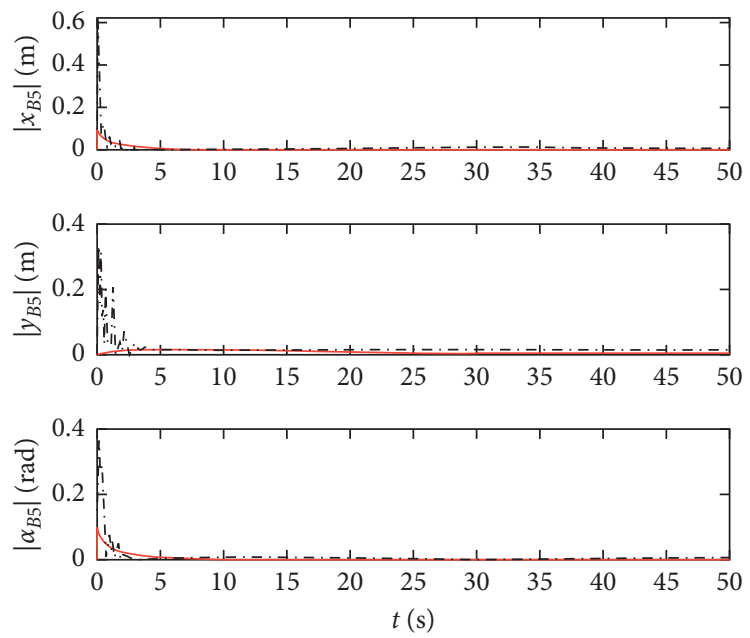

Data-driven RDMPC

-.. DMPC

(e)

Figure 3: The absolute value of state error $X_{i}$ of each mobile robot: (a) Robot 1; (b) Robot 2; (c) Robot 3; (d) Robot 4; (e) Robot 5 . 

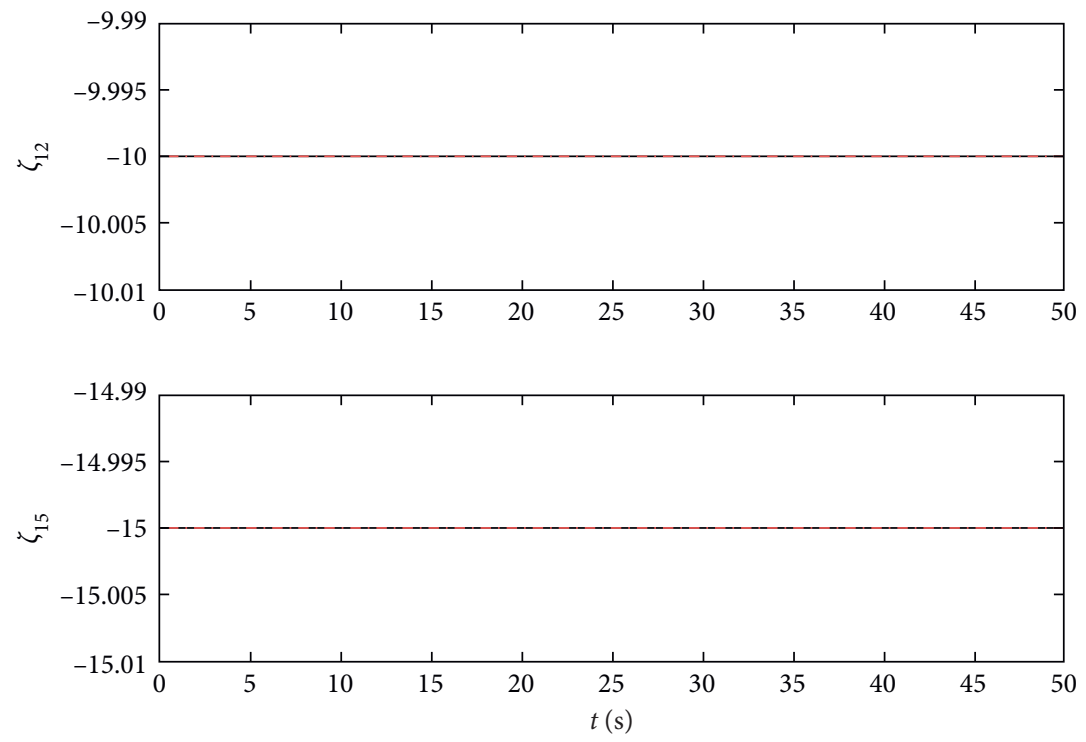

- Actual value

Desired value

(a)
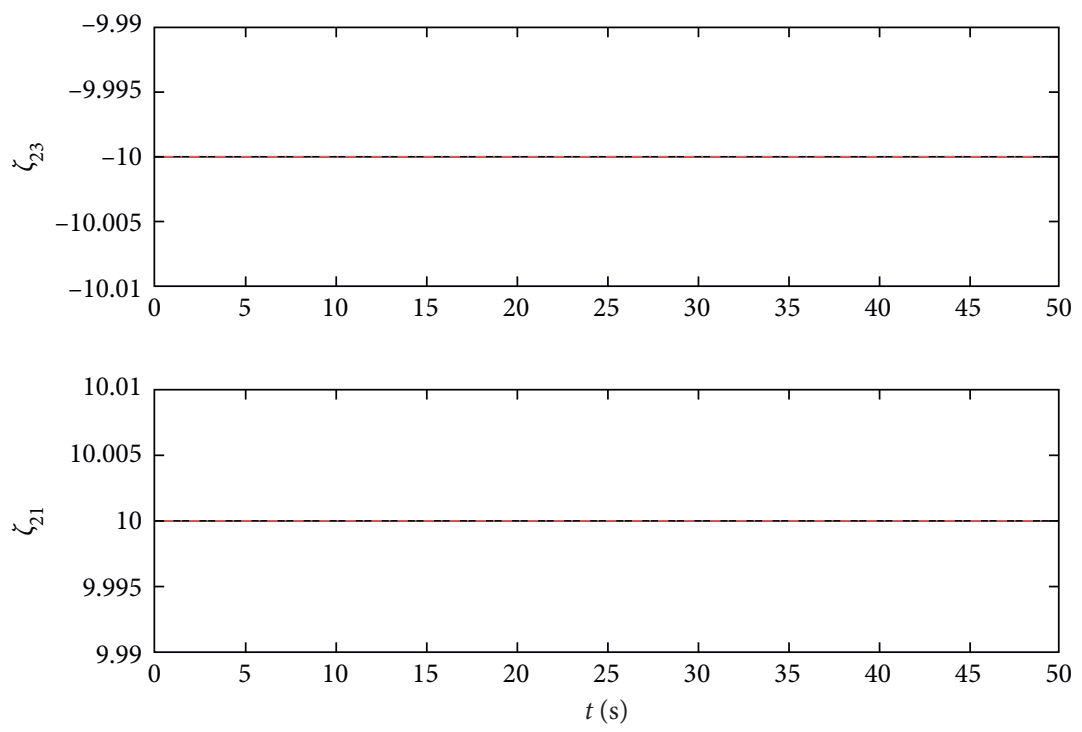

- Actual value

-... Desired value

(b)

Figure 4: Continued. 

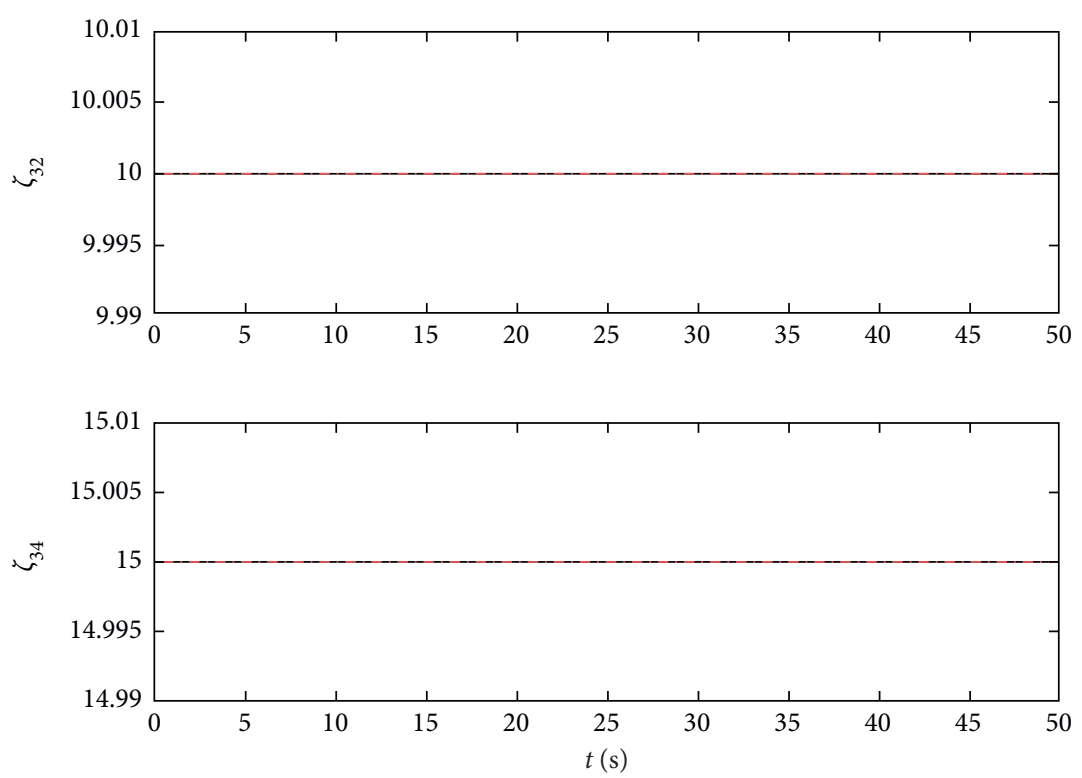

- Actual value

Desired value

(c)
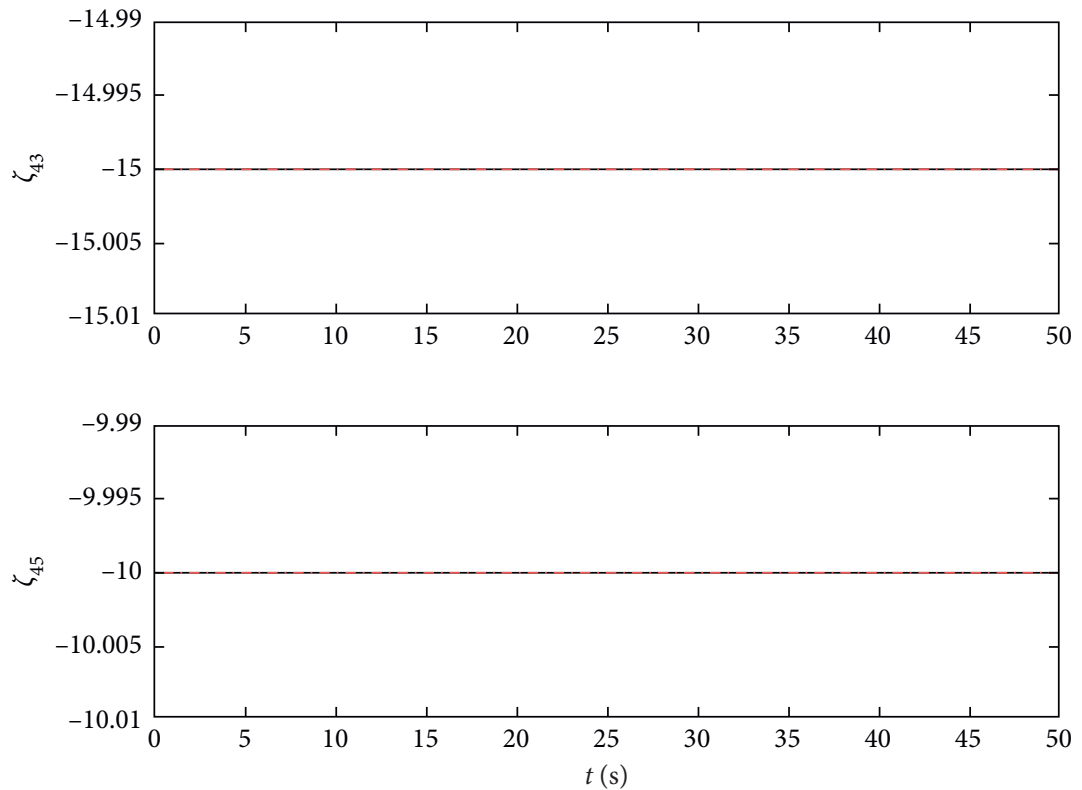

- Actual value

Desired value

(d)

FIgURe 4: Continued. 

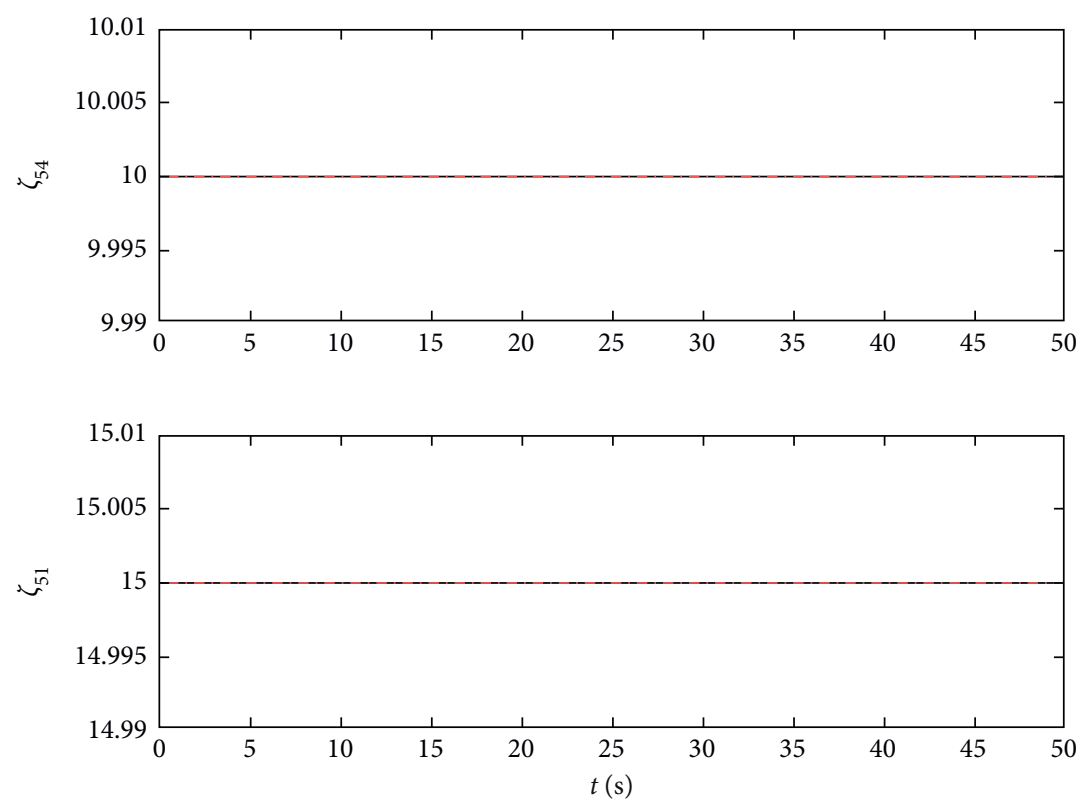

- Actual value

-... Desired value

(e)

FIgURE 4: The trajectory parameter difference $\zeta_{i j}$ and the desired trajectory parameter difference $\zeta_{i j}^{r}$ of each mobile robot: (a) Robot 1 ; (b) Robot 2; (c) Robot 3; (d) Robot 4; (e) Robot 5.
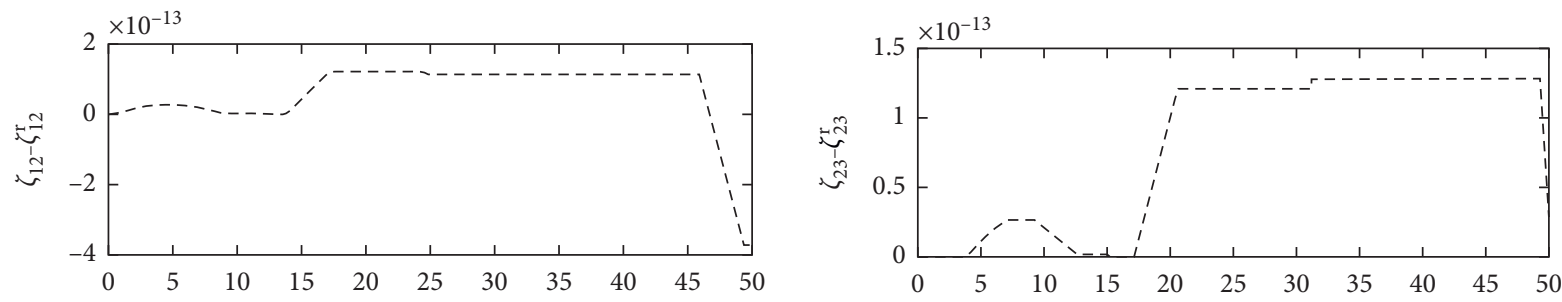

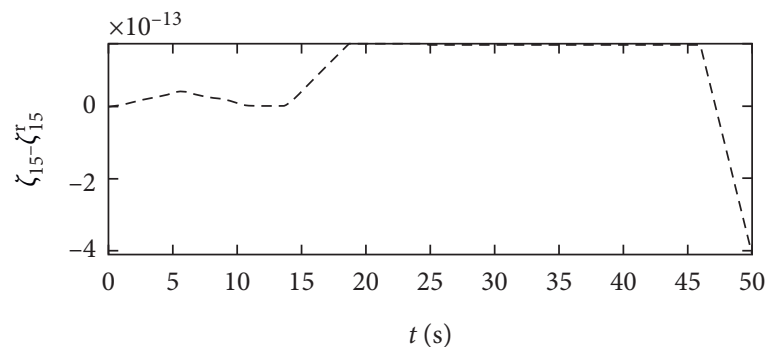

(a)

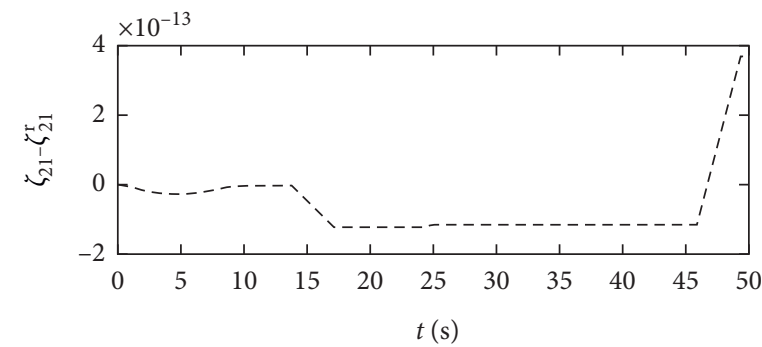

(b)

Figure 5: Continued. 

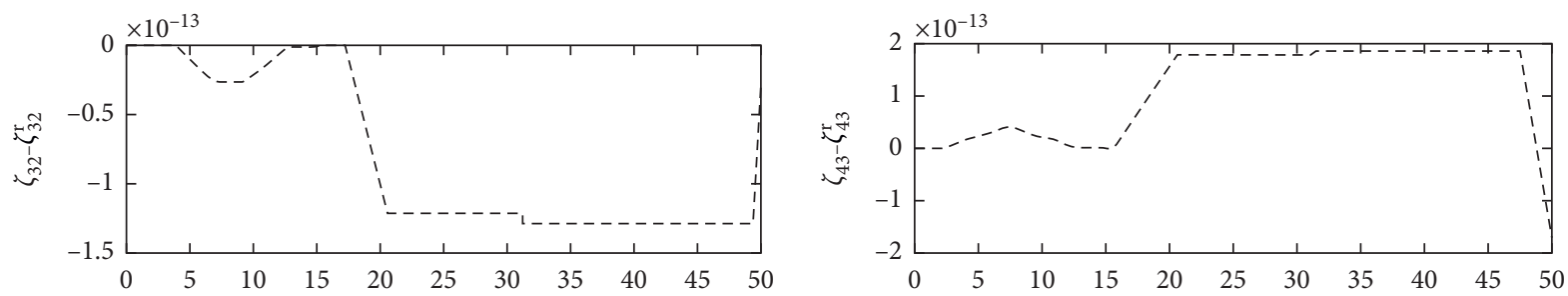

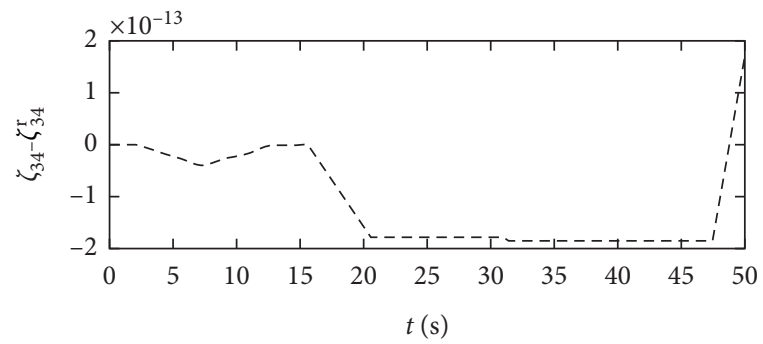

(c)

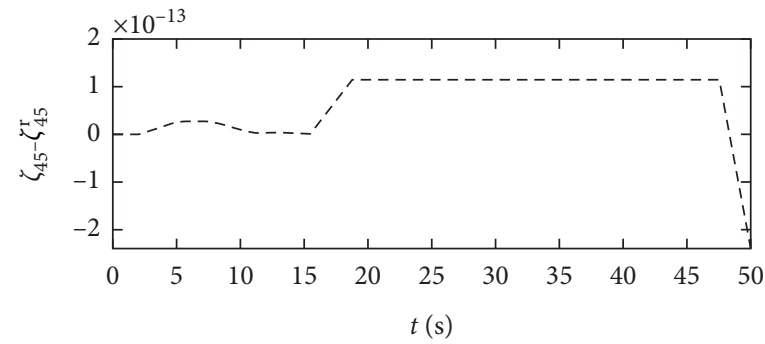

(d)
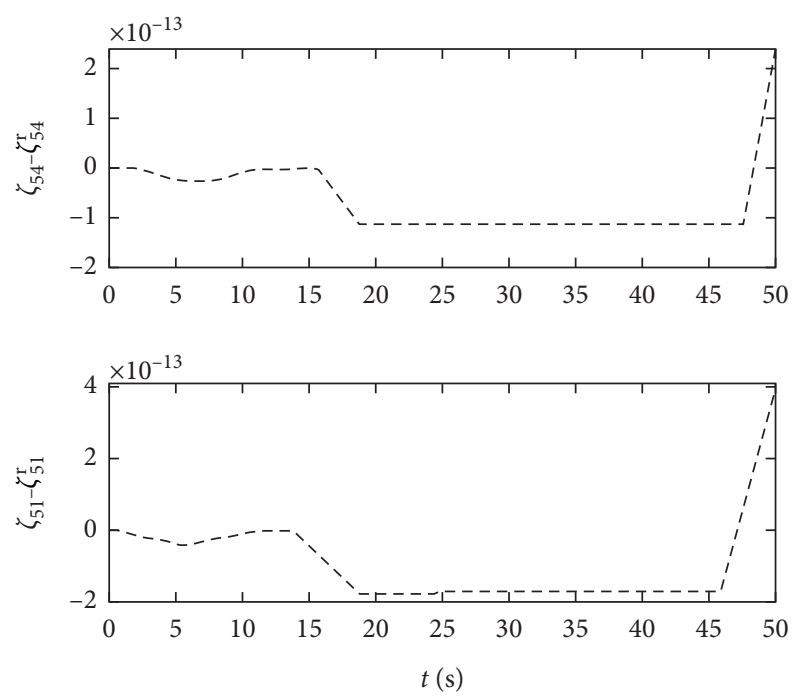

(e)

Figure 5: The different values between $\zeta_{i j}$ and $\zeta_{i j}^{r}$ of each mobile robot: (a) Robot 1; (b) Robot 2; (c) Robot 3; (d) Robot 4; (e) Robot 5. 

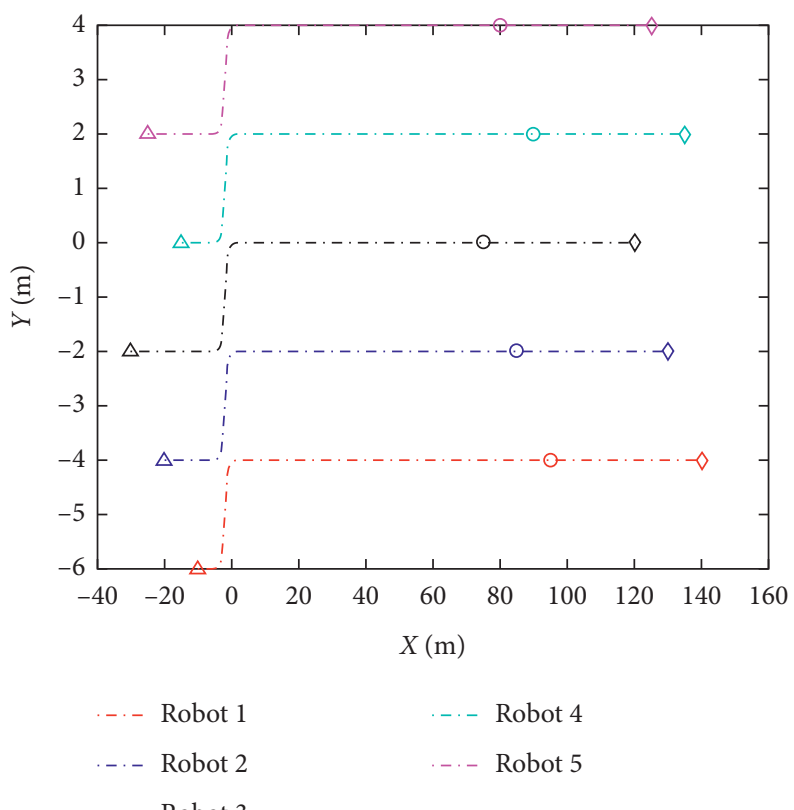

-..- Robot 3

Figure 6: Trajectories of five mobile robots.
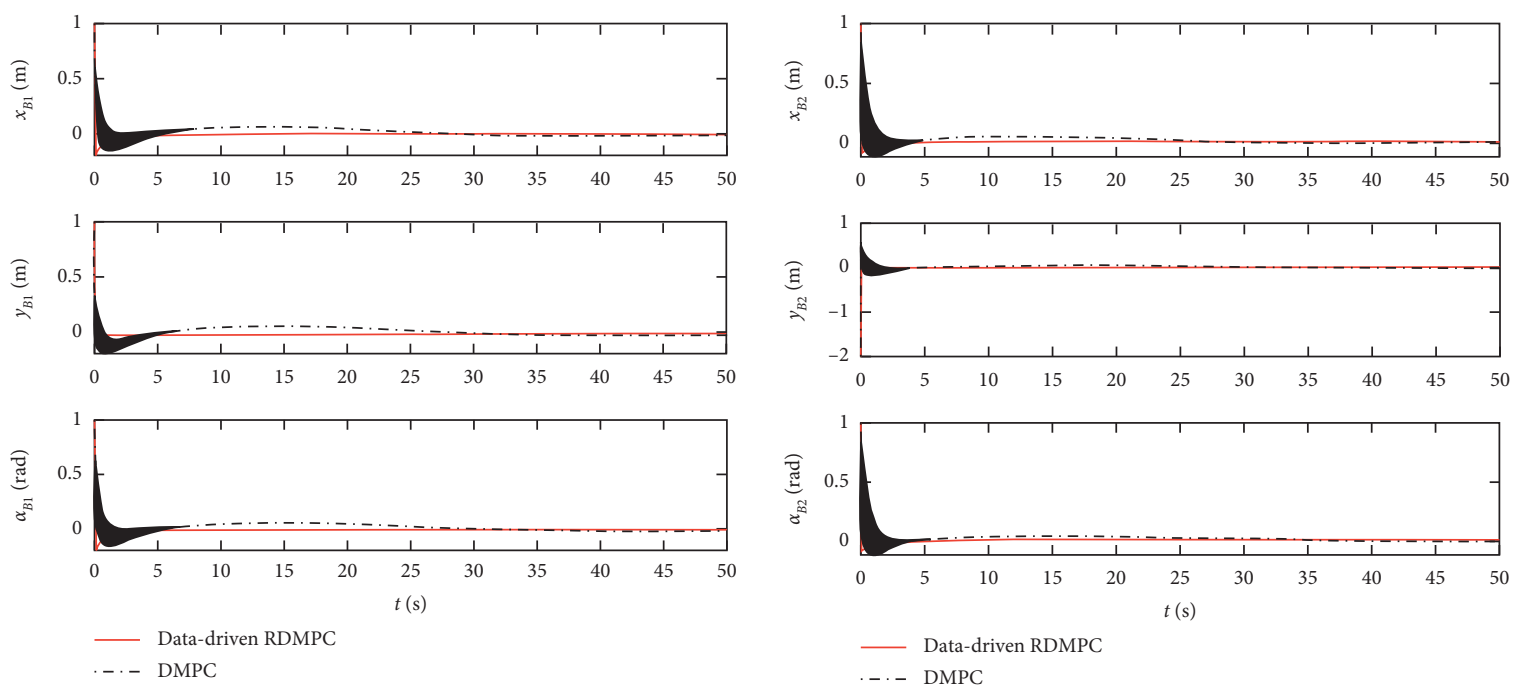

(a)

(b)

FIgURE 7: Continued. 

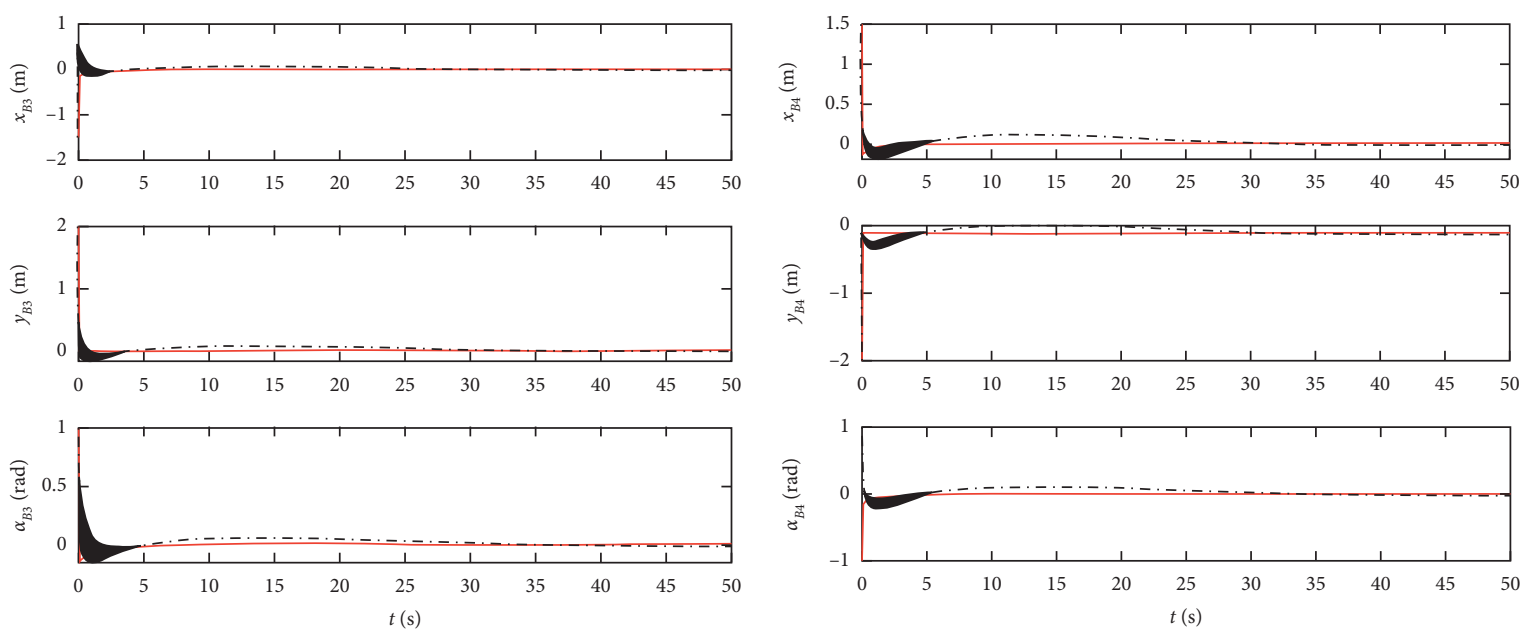

— Data-driven RDMPC

- . - DMPC

_ Data-driven RDMPC

-. - DMPC

(c)

(d)
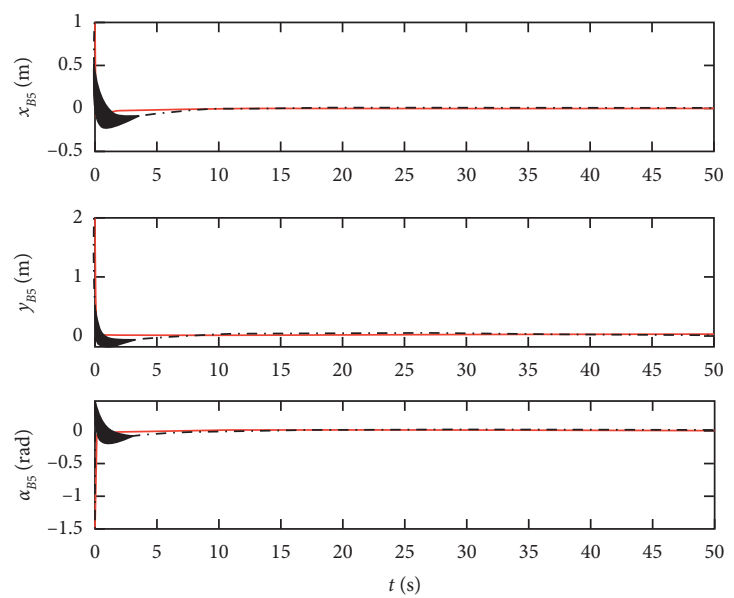

— Data-driven RDMPC

... - DMPC

(e)

Figure 7: The state error $X_{i}$ of each mobile robot: (a) Robot 1; (b) Robot 2; (c) Robot 3; (d) Robot 4; (e) Robot 5.
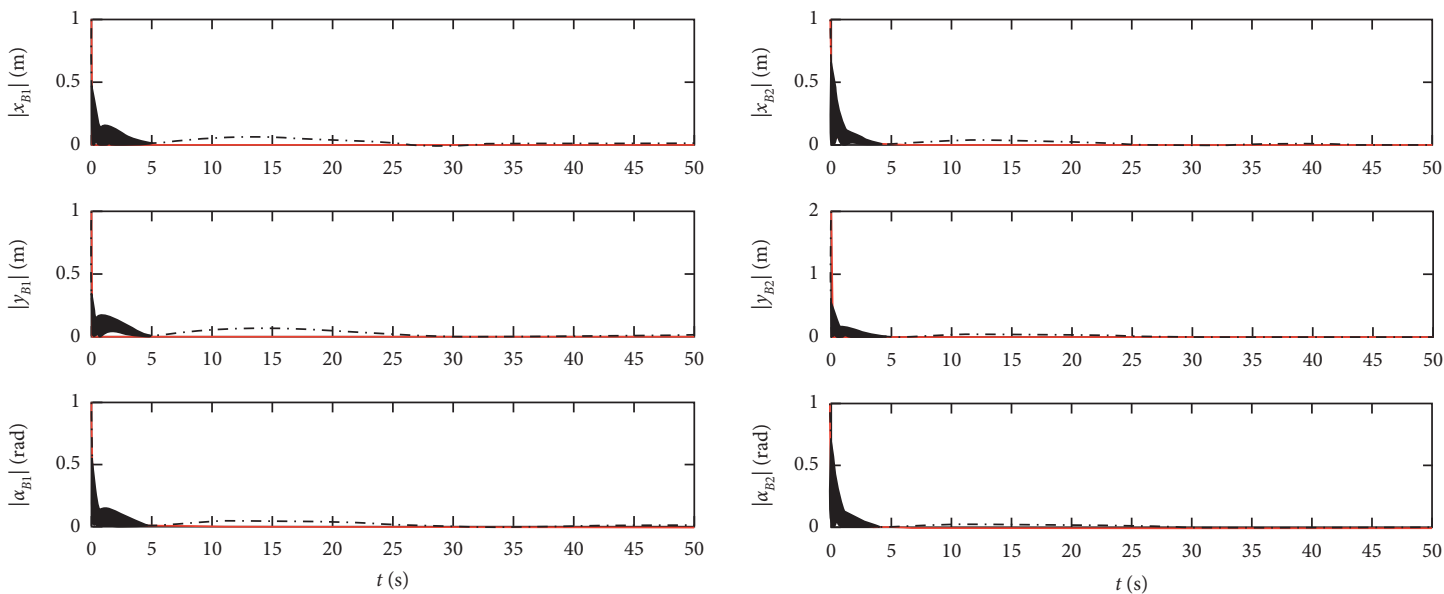

Data-driver

— Data-driven RDMPC

... DMPC

(a)

Figure 8: Continued. 

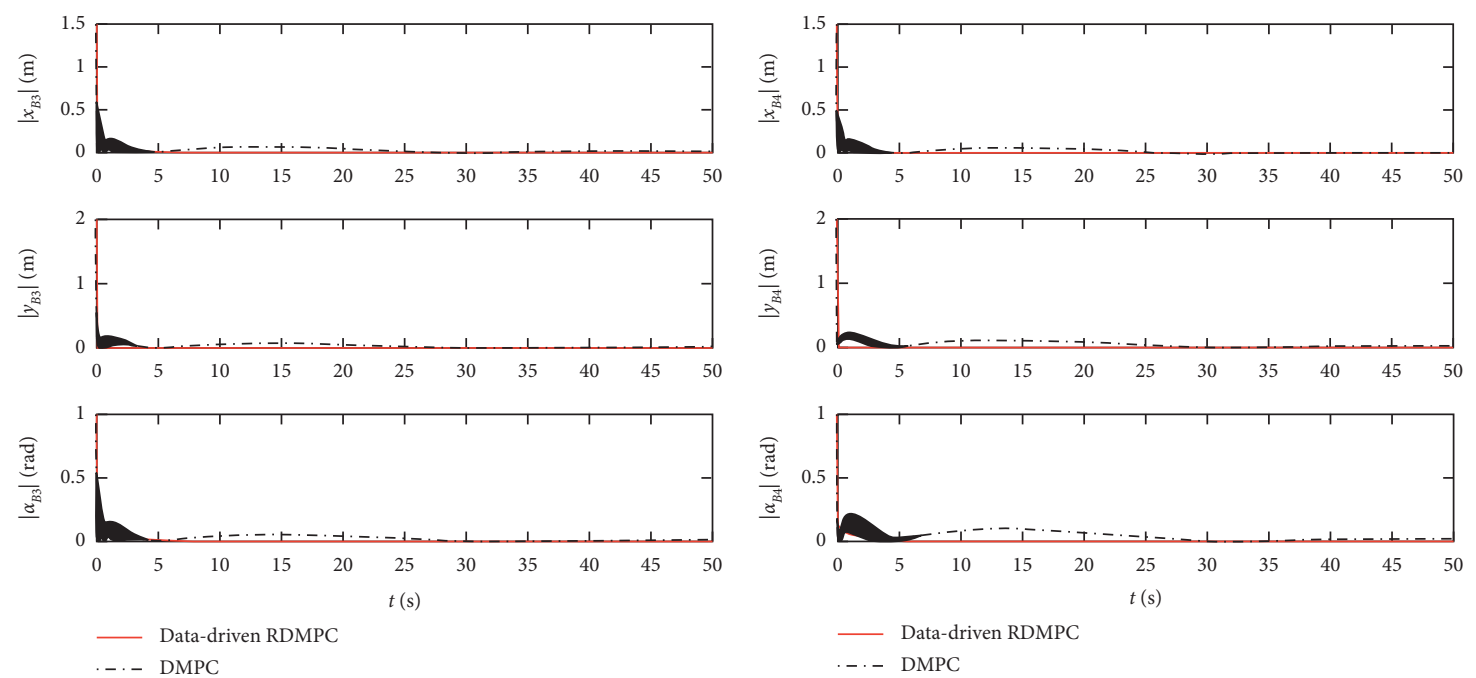

(c)

(d)
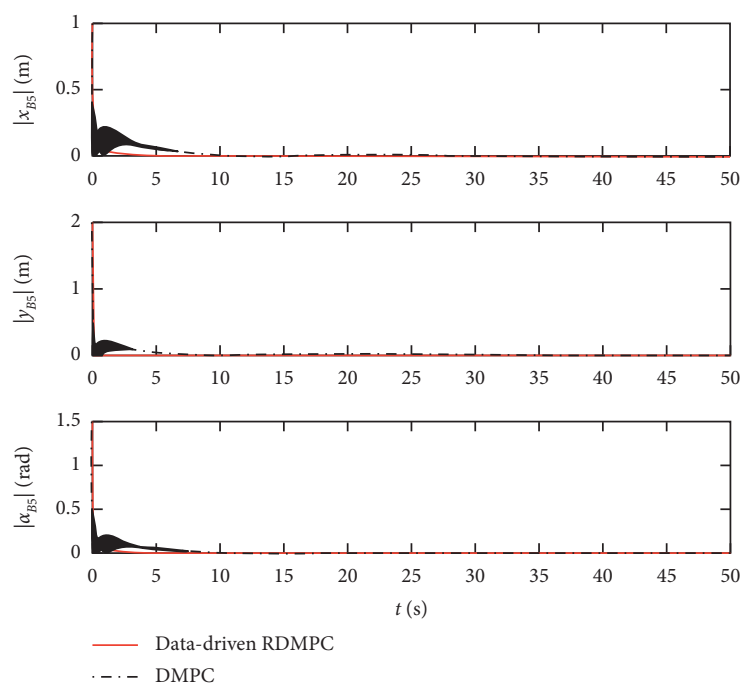

(e)

FIgURE 8: The absolute value of state error $X_{i}$ of each mobile robot: (a) Robot 1; (b) Robot 2; (c) Robot 3; (d) Robot 4; (e) Robot 5.
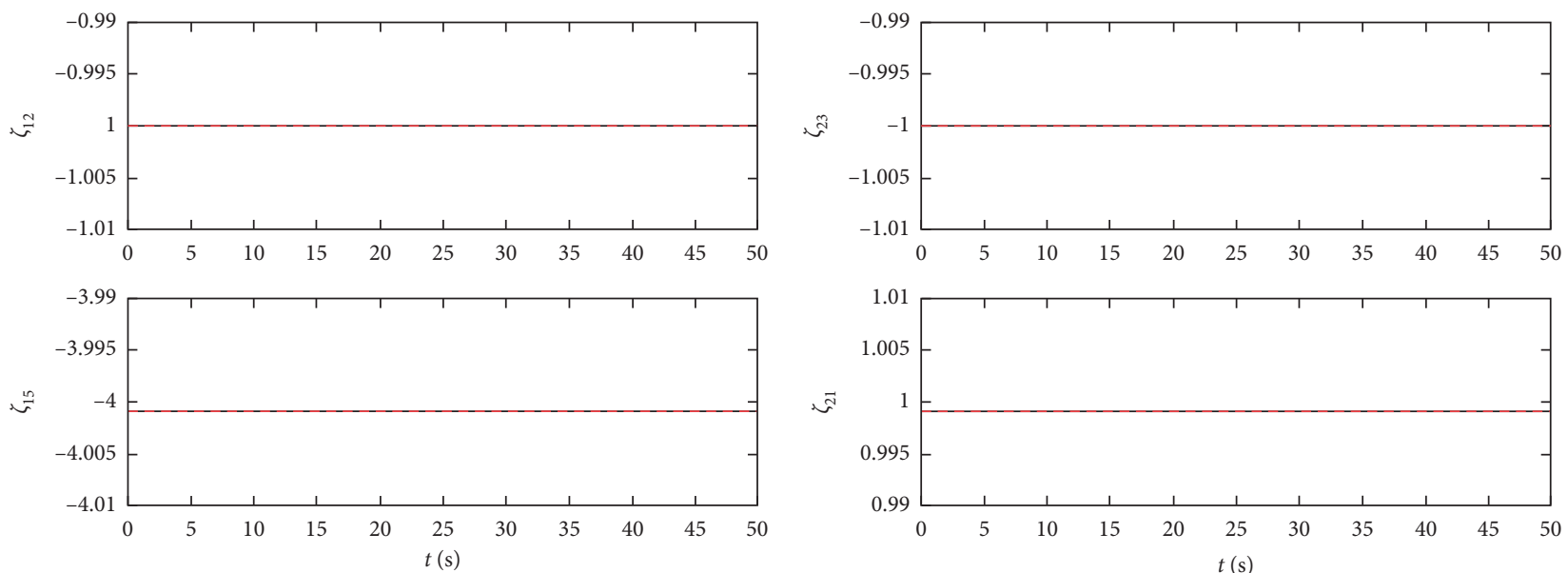

- Actual value

- Actual value

- - - Desired value

- - - Desired value

(a)

Figure 9: Continued. 

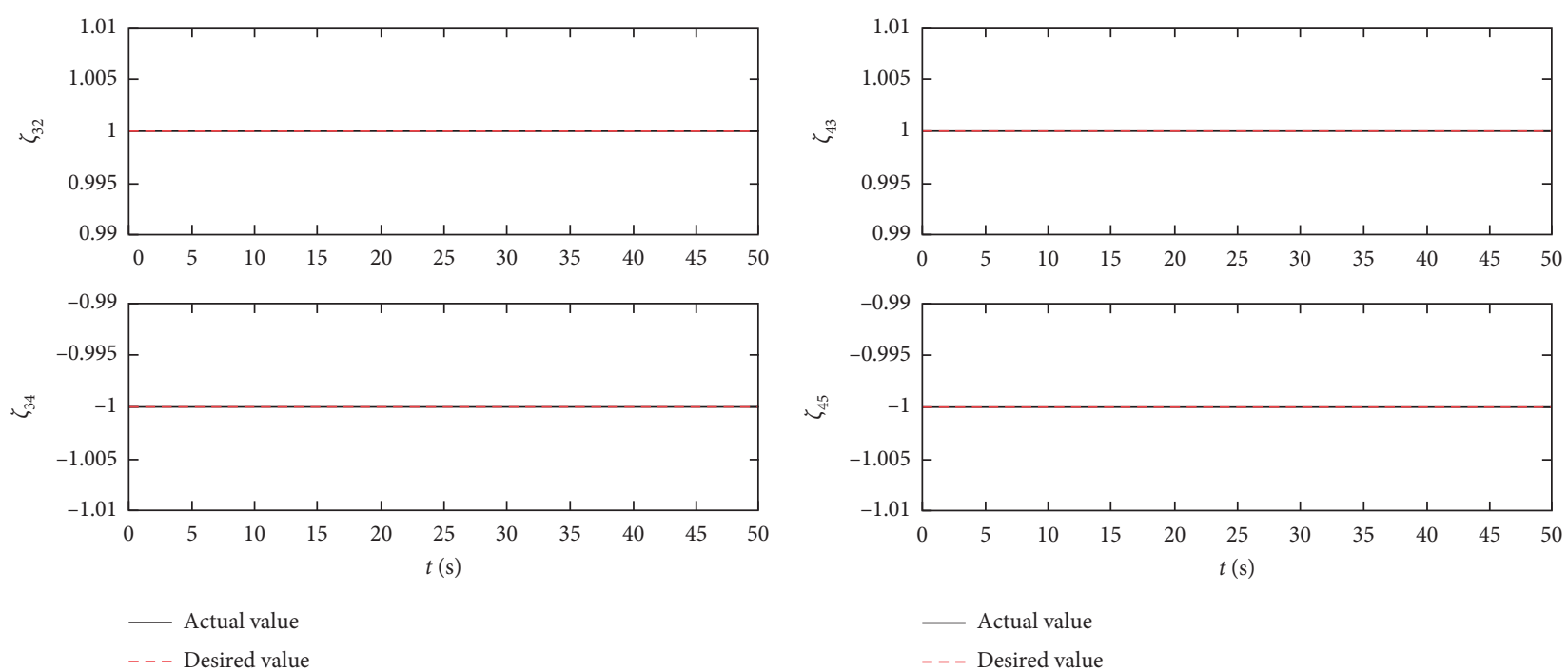

- Actual value

- - - Desired value

(c)

(d)
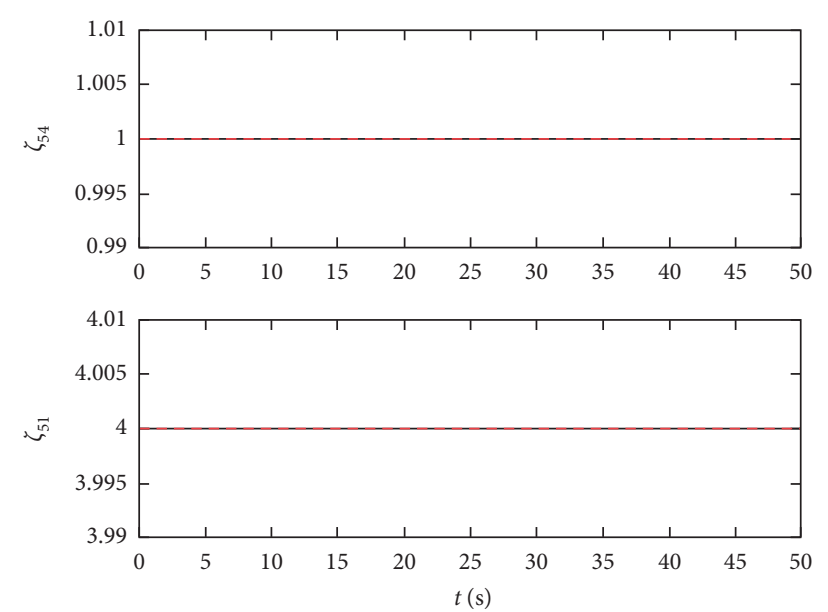

- Actual value

- - - Desired value

(e)

FIgURe 9: The trajectory parameter difference $\zeta_{i j}$ and the desired trajectory parameter difference $\zeta_{i j}^{r}$ of each mobile robot: (a) Robot 1 ; (b) Robot 2; (c) Robot 3; (d) Robot 4; (e) Robot 5. 

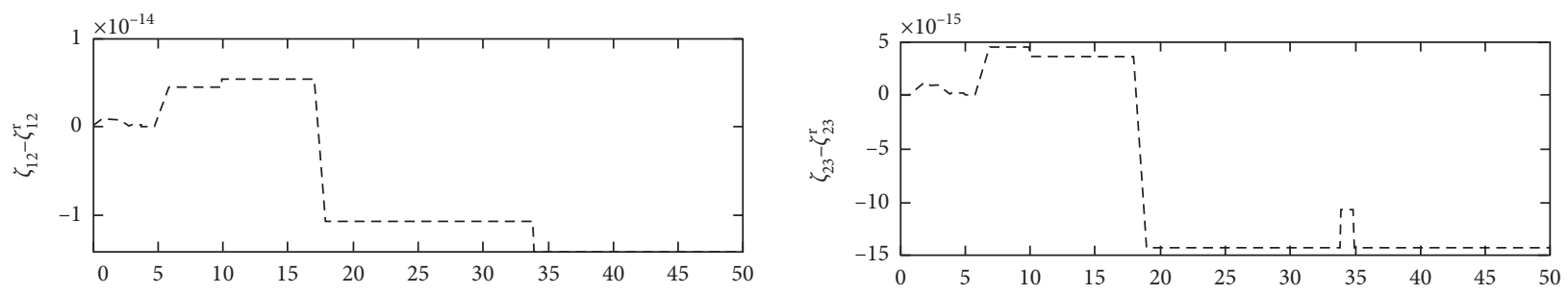

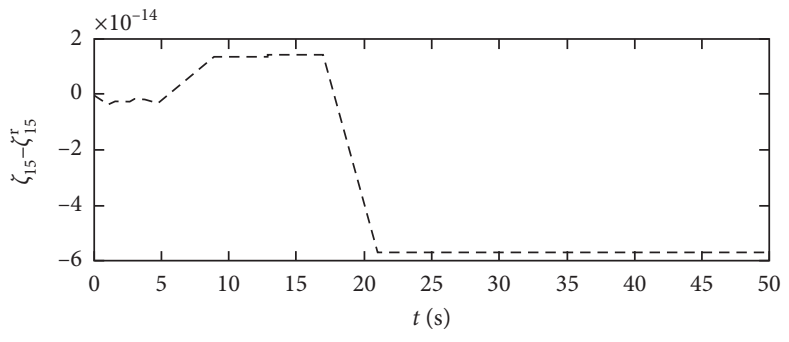

(a)
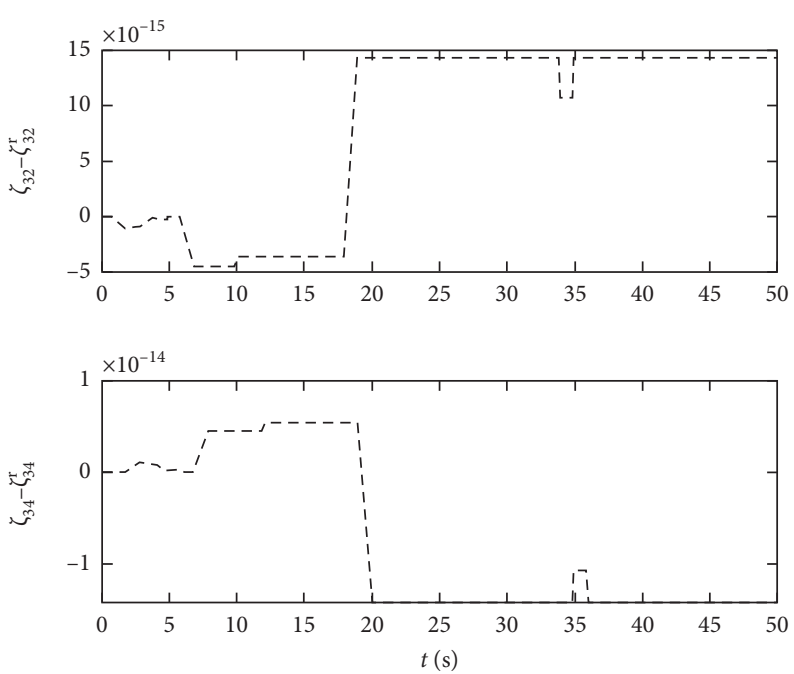

(c)

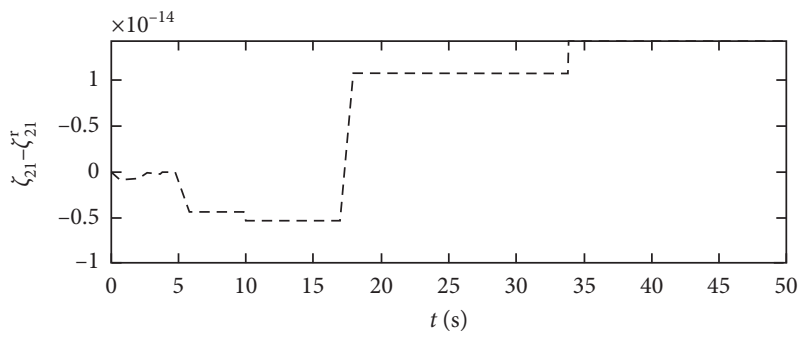

(b)
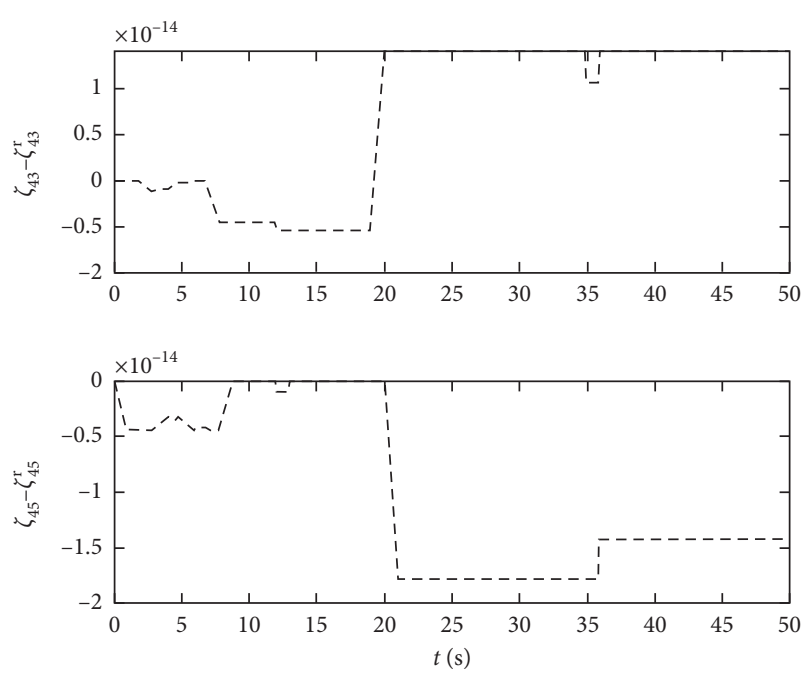

(d)
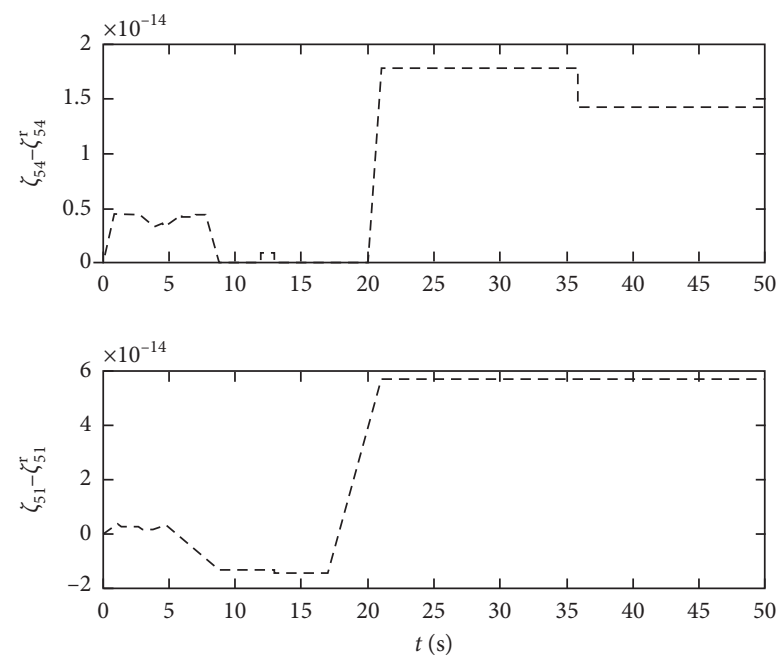

(e)

Figure 10: The different values between $\zeta_{i j}$ and $\zeta_{i j}^{r}$ of each mobile robot: (a) Robot 1; (b) Robot 2; (c) Robot 3; (d) Robot 4; (e) Robot 5. 


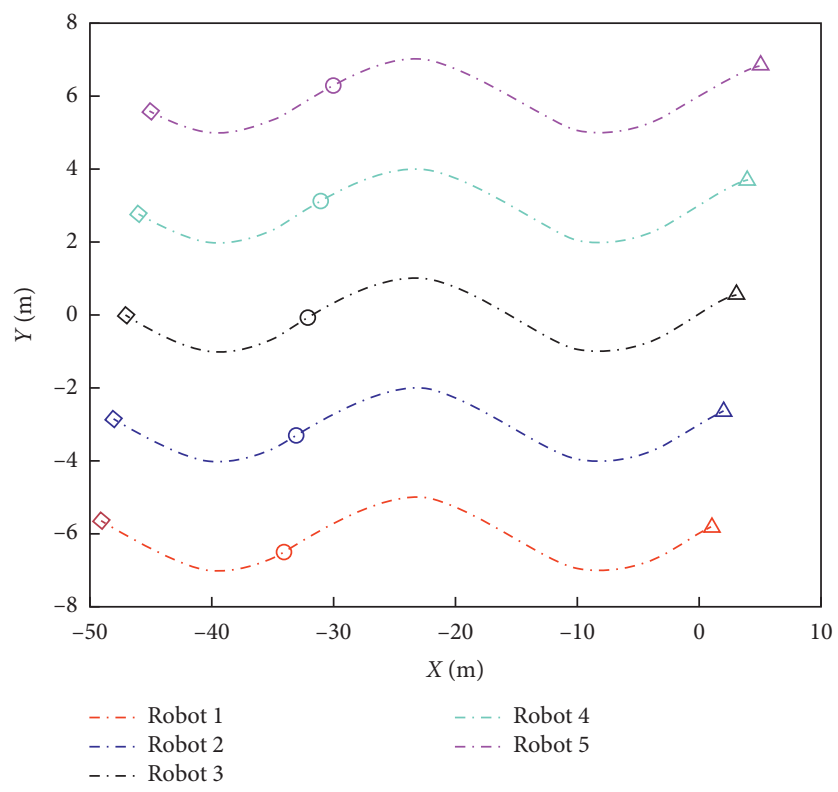

Figure 11: Trajectories of five mobile robots.

\section{Conclusions}

The traditional distributed model predictive control has been widely used in the field of control; however, it is difficult to handle the model uncertainty of the system. This paper discusses a data-driven robust distributed model predictive control with the model uncertainty. The accuracy of the algorithm is improved by applying the robust performance objective minimization to replace the quadratic performance objective minimization when establishing the optimization problem with considering the model uncertainty of system. And on this basis, the fluctuation of convergence curve and the computational efficiency are abated due to the way of substituting for the iterative algorithm with optimal solution. The system conservatism is reduced after the data-driven strategy that the future predictive value is represented by employing the linear law of historical data which is put to use in deriving the control policy. The stability of the system is proved by Lyapunov theorem, and the effectiveness of this method is illustrated by a set of simulation examples.

\section{Data Availability}

The data used to support this study are available from the corresponding authors upon request.

\section{Conflicts of Interest}

The authors declare that they have no conflicts of interest.

\section{Acknowledgments}

This research was funded by the Collaborative Innovation Center of Intelligent Green Manufacturing Technology and Equipment, grant number IGSD-2020-007; the Natural Science Foundation, grant number 5207053225; and the
Department of Science and Technology of Hubei Province, grant number 2020BIB012.

\section{References}

[1] N. Nigam, S. Bieniawski, I. Kroo, and J. Vian, "Control of multiple UAVs for persistent surveillance: algorithm and flight test results," IEEE Transactions on Control Systems Technology, vol. 20, no. 5, pp. 1236-1251, 2012.

[2] Q. Jiang and V. Kumar, "The inverse kinematics of cooperative transport with multiple aerial robots," IEEE Transactions on Robotics, vol. 29, no. 1, pp. 136-145, 2013.

[3] Z. Li, C. Yang, C. Y. Su, J. Deng, and W. Zhang, "Vision-based model predictive control for steering of a nonholonomic mobile robot," IEEE Transactions on Control Systems Technology, vol. 24, no. 2, pp. 553-564, 2016.

[4] K. Fan, Z. Li, H. Xiao, and X. Zhang, "Visual servoing of constrained mobile robots based on model predictive control," IEEE Transactions on Systems Man \& Cybernetics Systems, vol. 47, no. 99, pp. 1428-1438, 2016.

[5] H. Cai and G. Hu, "Distributed tracking control of an interconnected leader-follower multiagent system," IEEE Transactions on Automatic Control, vol. 62, no. 7, 2017.

[6] S. Khodabandeh, H. K. Shishavan, and F. Hashemzadeh, "Distributed fault tolerant control and estimation for leaderfollower consensus of multi-agent systems with actuator faults," in Proceedings of the IEEE 4th International Conference on Knowledge-Based Engineering and Innovation (KBEI), December 2017.

[7] N. Chen, Y. Wang, and L. Jia, "Multi - mobile robot leader -follower formation distributed control under switching topology," in Proceedings of the 2020 Chinese Control and Decision Conference (CCDC), pp. 2673-2678, Hefei, China, August 2020.

[8] X. Li, S. Xu, H. Gao, and H. Cai, "Distributed tracking of leader-follower multiagent systems subject to disturbed leader's information," IEEE Access, vol. 8, pp. 227970-227981, 2020. 
[9] S. Nantogma and H. Xiaoqin, "Behavior-based Genetic Fuzzy Control System for Multiple USVs Cooperative Target Protection," in Proceedings of the International Symposium on Autonomous Systems, University of Electronic Science and Technology, Chengdu, Sichuan, 2017.

[10] M. Agung Nursyeha, M. Rivai, and D. Purwanto, "LiDAR equipped robot navigation on behavior-based formation control for gas leak localization," in Proceedings of the 2020 International Seminar on Intelligent Technology and Its Applications (ISITIA), Surabaya, Indonesia, July 2020.

[11] H. Liang, Y. Fu, F. Kang, J. Gao, and N. Qiang, "A behaviordriven coordination control framework for target hunting by UUV intelligent swarm," IEEE Access, vol. 8, pp. 4838-4859, 2020.

[12] Y. Qingkai, C. Ming, F. Hao, C. Jie, and H. Jie, "Distributed formation stabilization for mobile agents using virtual tensegrity structures," in Proceedings of the 34th Chinese Control Conference (CCC), pp. 447-452, Hangzhou, China, July 2015.

[13] E. Abbasi, M. Ghayour, M. Danesh, P. Amiri, and M. H. Yoosefian, "Formation flight control and path tracking of a multi-quadrotor system in the presence of measurement noise and disturbances," in Proceedings of the 2018 6th RSI International Conference on Robotics and Mechatronics (IcRoM), pp. 273-279, Tehran, Iran, October 2018.

[14] H. Wei, Q. Sun, J. Chen, and Y. Shi, "Robust distributed model predictive platooning control for heterogeneous autonomous surface vehicles," Control Engineering Practice, vol. 107, Article ID 104655, 2021.

[15] H. Wang, J. Jiang, W. Wu, L. Liu, D. Wang, and Z. Peng, "Robust distributed guidance and control of multiple autonomous surface vehicles based on extended state observers and finite-set model predictive control," in Proceedings of the 2020 5th International Conference on Automation, Control and Robotics Engineering (CACRE), pp. 235-239, Dalian, China, September 2020.

[16] S. J. Yoo and B. S. Park, "Connectivity-preserving approach for distributed adaptive synchronized tracking of networked uncertain nonholonomic mobile robots," IEEE Transactions on Cybernetics, vol. 48, no. 9, pp. 2598-2608, 2018.

[17] A. Marino, "Distributed adaptive control of networked cooperative mobile manipulators," IEEE Transactions on Control Systems Technology, vol. 26, no. 5, pp. 1646-1660, 2018.

[18] S. Liu, Q. Fei, W. Bo, and Q. Geng, "Distributed adaptive integral sliding mode attitude consensus control for multiple autonomous underwater vehicles," in Proceedings of the 2018 37th Chinese Control Conference (CCC), Wuhan, China, July 2018.

[19] C. Deng, W. Gao, and W. Che, "Distributed adaptive faulttolerant output regulation of heterogeneous multi-agent systems with coupling uncertainties and actuator faults," IEEE/CAA Journal of Automatica Sinica, vol. 7, no. 4, pp. 1098-1106, 2020.

[20] B. Cheng and Z. Li, "Designing fully distributed adaptive event-triggered controllers for networked linear systems with matched uncertainties," IEEE Transactions on Neural Networks and Learning Systems, vol. 30, no. 12, pp. 1-11, 2018.

[21] S. Islam, T. A. Khawli, A. Alzaabi, and A. Sunda-Meya, "Consensus based distributed robust adaptive control for second-order nonlinear multi-agent systems with uncertainty," in Proceedings of the 2018 IEEE International Conference on Systems, Man, and Cybernetics (SMC), October 2018.

[22] S. Islam, P. X. Liu, and A. E. Saddik, "Consensus based distributed cooperative control for multiple miniature aerial vehicles with uncertainty," in Proceedings of the IEEE International Conference on Systems, October 2017.

[23] D. D. Santana, M. Martins, and D. Odloak, "An efficient cooperative-distributed model predictive controller with stability and feasibility guarantees for constrained linear systems," Systems \& Control Letters, vol. 141, Article ID 104701, 2020.

[24] C. F. Hu, Z. Meng, G. Qu, H. S. Shin, and A. Tsourdos, "Distributed cooperative path planning for tracking ground moving target by multiple fixed-wing UAVs via DMPC-GVD in urban environment," International Journal of Control, Automation and Systems, vol. 19, pp. 823-836, 2020.

[25] L. Chen, H. Hopman, and R. R. Negenborn, "Distributed model predictive control for vessel train formations of cooperative multi-vessel systems," Transportation Research Part C: Emerging Technologies, vol. 92, pp. 101-118, 2018.

[26] H. Ebel, E. S. Ardakani, and P. Eberhard, "Distributed model predictive formation control with discretization-free path planning for transporting a load," Robotics and Autonomous Systems, vol. 96, 2017.

[27] N. A. Mardi, Data-driven subspace-based model predictive control, Ph.D. Dissertation, RMIT University, Melbourne, Australia, 2010.

[28] Y. K. Lv and R. H. Chi, "Data-driven adaptive iterative learning predictive control," in Proceedings of the IEEE 2017 6th Data Driven Control and Learning Systems (DDCLS), pp. 374-377, Chongqing, China, May 2017.

[29] A. Liniger, X. Zhang, P. Aeschbach, A. Georghiou, and J. Lygeros, "Racing miniature cars: enhancing performance using Stochastic MPC and disturbance feedback," in Proceedings of the 2017 American Control Conference (ACC), pp. 5642-5647, Seattle, WA, USA, May 2017.

[30] F. W. Yang and J. Chen, "Data-driven subspace-based adaptive fault detection for solar power generation systems," IET Control Theory \& Applications, vol. 7, no. 11, pp. 14981508, 2013.

[31] Y. Han and Q. Zhu, "Robust optimal control of omni-directional mobile robot using model predictive control method," in Proceedings of the 2019 Chinese Control Conference (CCC), pp. 4679-4684, Guangzhou, China, July 2019.

[32] L. Zhang, X. Wei, L. Bo, and Z. He, "Synthesis of distributed MPC with structured uncertainties," in Proceedings of the 2016 35th Chinese Control Conference (CCC), pp. 4360-4365, Chengdu, China, July 2016.

[33] S. Hui, M. Wang, and Y. Han, "Research on adaptive algorithm for weight partial update of ANC," in Proceedings of the IEEE 2nd International Conference on Information Communication and Signal Processing (ICICSP), pp. 121-125, Weihai, China, September 2019.

[34] M. ÖZCAN and F. Aliew, "Review of iterative numerical methods preferred in technical application to increase efficiency," in Proceedings of the 2020 IEEE Ukrainian Microwave Week (UkrMW), pp. 874-879, Kharkiv, Ukraine, September 2020.

[35] L. Liu, J. Li, Y. Weng, N. Wang, and Y. Liu, "Data-driven sliding mode control with moving surface for unknown MIMO discrete-time nonlinear processes," in Proceedings of the 2019 IEEE 8th Data Driven Control and Learning Systems Conference (DDCLS), pp. 1253-1257, Dali, China, May 2019.

[36] L. Shi, H. Teng, X. Kan, and K. Karydis, "A data-driven hierarchical control structure for systems with uncertainty," in Proceedings of the 2020 IEEE Conference on Control Technology and Applications (CCTA), pp. 57-63, Montreal, Canada, September 2020. 
[37] Z. Zheng and H. Yi, "Backstepping control design for UAV formation with input saturation constraint and model uncertainty," in Proceedings of the 2017 36th Chinese Control Conference (CCC), pp. 6056-6060, Dalian, China, July 2017.

[38] J. Sun, J. Yi, and Z. Pu, "Fixed-Time adaptive fuzzy control for uncertain nonstrict-feedback systems with time-varying constraints and input saturations," IEEE Transactions on Fuzzy Systems, vol. 99, 2021. 